Document downloaded from:

http://hdl.handle.net/10251/122878

This paper must be cited as:

Piqueras-García, MÁ.; Company Rossi, R.; Jódar Sánchez, LA. (2018). Numerical analysis and computing of free boundary problems for concrete carbonation chemical corrosion. Journal of Computational and Applied Mathematics. 336:297-316.

https://doi.org/10.1016/j.cam.2017.12.036

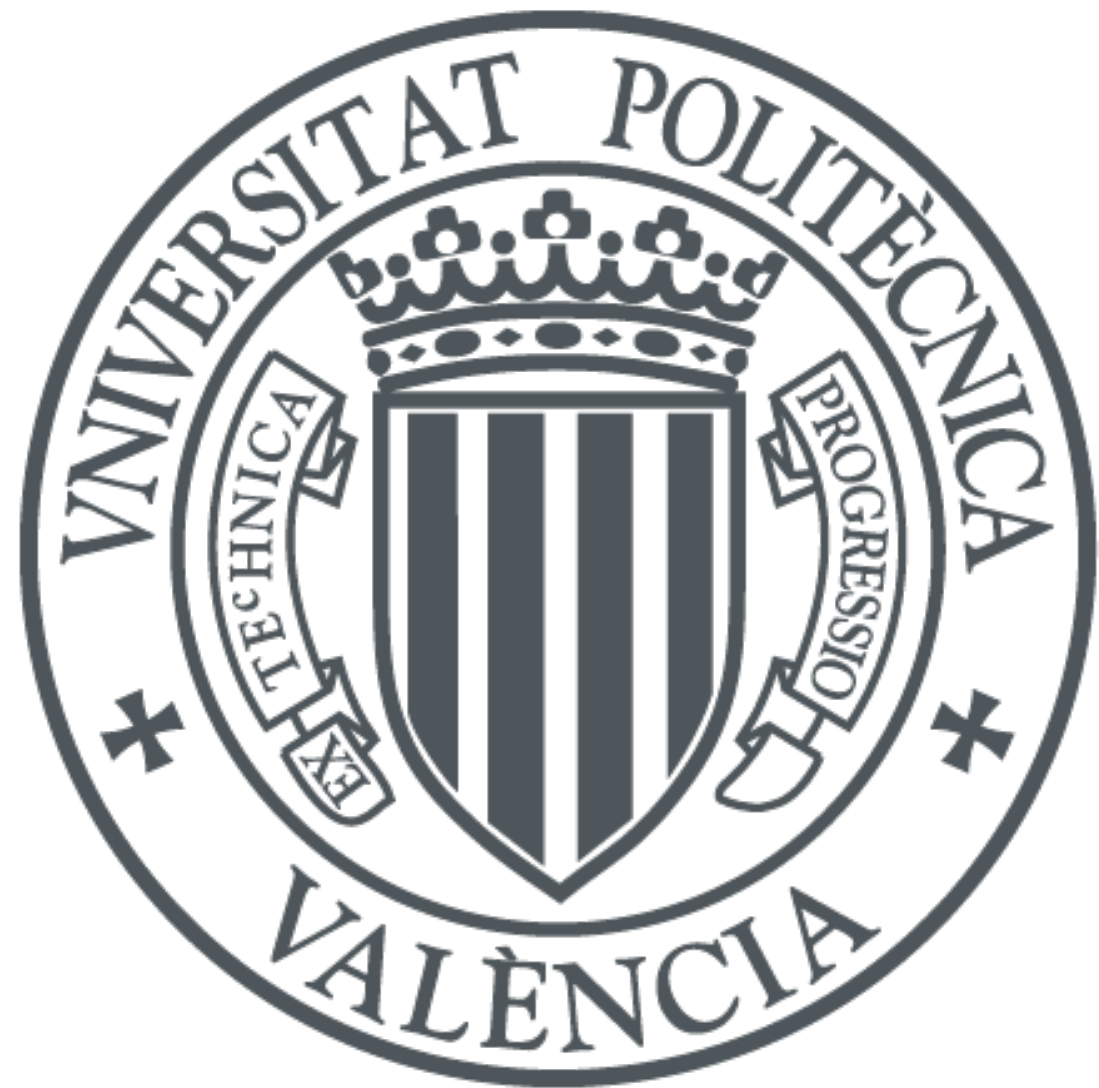

The final publication is available at

http://doi.org/10.1016/j.cam.2017.12.036

Copyright Elsevier

Additional Information 


\title{
Numerical analysis and computing of free boundary problems for concrete carbonation chemical corrosion
}

\author{
M.-A. Piqueras, R. Company ${ }^{1}$, L. Jódar \\ Instituto de Matemática Multidisciplinar, Universitat Politècnica de València, Camino de \\ Vera $s / n$, 46022 Valencia, Spain
}

\begin{abstract}
This paper deals with the construction, analysis and computation of a numerical method to solve a moving boundary coupled nonlinear system of parabolic reaction-diffusion equations, arising in concrete carbonation problems. By means of a front-fixing transformation, the domain of the problem becomes fixed, and the position of the moving carbonation front has to be determined together with the mass concentrations of the involved chemical species. Qualitative properties like positivity and stability of the numerical solution are established. Spatial monotone behaviour of the solution is also proved. Numerical examples illustrate these results.

Keywords: Concrete carbonation chemical corrosion, Free boundary problems, Nonlinear PDE system, Numerical analysis, Finite difference methods.
\end{abstract}

\section{Introduction}

The effect of concrete carbonation in buildings and civil works is a relevant issue in civil engineering construction and architecture due to the deterioration

\footnotetext{
Email addresses: mipigar@cam.upv.es (M.-A. Piqueras), rcompany@imm.upv.es (R. Company), ljodar@imm.upv.es (L. Jódar)

${ }^{1}$ Corresponding author.
} 
of building materials and its potential incidence in the citizens safety.

$5 \quad$ This matter is also related to environment, public health and ecology because the carbonation effect is in part due to the influence of traffic and industries. This problem may be regarded as a particular case inside of the wide area of materials corrosion. Apart from the concrete carbonation, other damaging processes are those related to the effects of sulfuric acid attack, [6], or chloride penetration in concrete structures, 20, 25].

The carbonation of concrete is a natural phenomenon that affects the performance, serviceability and safety of concrete structures. The diffusion of the environmental carbon dioxide in the dry parts and its reaction in the unsaturated concrete pores, cause a drop in $p H$ of the alkaline components near the 15 steel bars, and the oxide film around the steel surface declines its ability to protect bars from corrosion. Thus, carbonation process leads to the corrosion of the embedded reinforcing bars, reducing the service life of concrete structures such as bridges, sewage pipes and seawalls. The annual carbon dioxide concentration growth rate has increased from 1.4 parts per million (ppm) per year during the period 1960-2005 to 1.9 ppm per year during the period 1995-2005. Following the current tendency, studies estimate that atmospheric $\mathrm{CO}_{2}$ concentration could increase from 379 ppm in 2005 over 1000 ppm by the year 2100, resulting in an increase of corrosion risk, see [1, 8].

The presence of carbon dioxide under normal atmospheric conditions and also emitted as industrial output, enters the gaseous phase of the pores. Carbon dioxide is transported by diffusion and dissolved in the aqueous phase, where it is further transported towards the place where carbonation reaction takes place. The other reactant, calcium hydroxide, is initially in the solid matrix of cement. By means of a dissolution process, it arrives in the aqueous phase of the 30 pores and reacts with $\mathrm{CO}_{2}$ present in the aquaeus phase. The reaction products are water together with calcium carbonate. Several experimental studies have addressed the problem of concrete carbonation, studying the behaviour of the 
position of the interface that separates the carbonated and non carbonated part, for large times, [3, $9,10,18,23,24]$.

The concrete carbonation depth needs to be estimated and there is need of models capable to predict the depth of $\mathrm{CO}_{2}$ penetration in concrete structures accurately, see [15]. In [15, 16, 17], the authors propose a moving interface model to forecast the position of the carbonation front and the profiles of the active concentrations, showing qualitative properties of the mass concentration 40 solutions, such as positivity and upper boundedness. Numerical simulations of the solution of the model using the finite element method are performed in [15, 16]. The model involves the unknown moving boundary and the concentrations of involved species by means of the solution of a coupled nonlinear system of parabolic reaction-diffusion equations. As the best model may be wasted 45 with a disregarded computation, its numerical analysis is necessary. Dealing with boundary-value problems whose boundary of the domain is not known in advance but has to be determined as part of the solution, the term "free boundary problem" is commonly used when the boundary is stationary and a steady-state problem exists. On the other hand, moving boundaries, like the problem studied in this paper, are associated with time-dependent problems. However it is usual to include both types of problems under the single term "free boundary problem" [4]. Recent works on numerical analysis of finite difference methods for free boundary problems can be found, for instance, in [21] for a two-phase Stefan problem of melting and in [19] for a moving boundary 55 problem in population dynamics.

Other recent different model of concrete carbonation phenomenon is exposed in [11], where an initial boundary value problem is used to study carbon dioxide transport in a bounded domain of $\mathbb{R}^{3}$.

The concrete element is supposed to be a sample with thickness equal to $L$. The space variable $x$ is measured from the exposed boundary $x=0$ to the sealed boundary $x=L$, and the unknown carbonation front or moving 
boundary is denoted by $x=S(t)$, satisfying $0<S(t)<L$. The whole domain $[0, L]$ is divided in two subdomains, the so called carbonated zone $\Omega_{1}(t)=$ $\left[0, S(t)\left[\right.\right.$ and uncarbonated zone $\left.\left.\Omega_{2}(t)=\right] S(t), L\right]$. The chemical species present in $\Omega_{1}(t)$ are $\mathrm{CO}_{2}(\mathrm{aq}), \mathrm{CO}_{2}(\mathrm{~g}), \mathrm{CaCO}_{3}(\mathrm{aq})$ and $\mathrm{H}_{2} \mathrm{O}$, and the species in $\Omega_{2}(t)$ are $\mathrm{Ca}(\mathrm{OH})_{2}(\mathrm{aq})$ and $\mathrm{H}_{2} \mathrm{O}$. Here, (aq) and (g) refer to species in aqueous and gaseous environments, respectively. $\mathrm{CaCO}_{3}(\mathrm{aq})$ is created in the carbonation front according to the reaction

$$
\mathrm{CO}_{2}(\mathrm{aq})+\mathrm{Ca}(\mathrm{OH})_{2}(\mathrm{aq}) \longrightarrow \mathrm{CaCO}_{3}(\mathrm{aq})+\mathrm{H}_{2} \mathrm{O}
$$

and at any time $t$ is uniformly distributed in $\Omega_{1}(t)=[0, S(t)[,[16]$.

The mass concentrations of the species are represented by the following variables, where time takes values in the interval $0 \leq t \leq T$,

$$
\begin{aligned}
& \bar{U}_{1}(x, t)=\left[\mathrm{CO}_{2}(a q)\right], \quad \bar{U}_{2}(x, t)=\left[\mathrm{CO}_{2}(g)\right], \quad \bar{U}_{5}(x, t)=\left[\mathrm{H}_{2} \mathrm{O}\right], \quad 0 \leq x \leq S(t), \\
& \bar{U}_{3}(x, t)=\left[\mathrm{Ca}(\mathrm{OH})_{2}(a q)\right], \quad \bar{U}_{6}(x, t)=\left[\mathrm{H}_{2} \mathrm{O}\right], \quad S(t) \leq x \leq L, \\
& \bar{U}_{4}(t)=\left[\mathrm{CaCO}_{3}(a q)\right] \text {, }
\end{aligned}
$$

60 where $T>0$ is the time horizon.

Throughout this work, we will use the notation $\bar{U}_{i}=\bar{U}_{i}(x, t), 1 \leq i \leq 6$, $i \neq 4$, and $\bar{U}_{4}=\bar{U}_{4}(t)$ for the unknown concentrations. Note that the unknown $\bar{U}_{4}$ depends only of the time because it refers to the concentration of $\mathrm{CaCO}_{3}(\mathrm{aq})$ at the unknown carbonation front $S(t)$.

The porosity of the concrete sample is given by the parameter $\phi$, while air and water fractions of the pores are denoted by $\phi_{\omega}$ and $\phi_{a}$, respectively. According to [5], it is assumed that the exchange of $\mathrm{CO}_{2}$ between the gas and liquid phases is modeled by linear Henry laws of the type

$$
f_{1, \text { Henry }}=-P_{1}\left(\phi \phi_{\omega} \bar{U}_{1}-Q_{1} \phi \phi_{a} \bar{U}_{2}\right), \quad f_{2, \text { Henry }}=P_{2}\left(\phi \phi_{\omega} \bar{U}_{1}-Q_{2} \phi \phi_{a} \bar{U}_{2}\right),
$$

${ }_{65}$ where $P_{1}, P_{2}$ are mass transfer parameters through the interface air-water and $Q_{1}, Q_{2}$ are the positive Henry-like constants. 
The dissolution rate for $\mathrm{Ca}(\mathrm{OH})_{2}(\mathrm{aq})$ is given by

$$
f_{\text {Diss }}=-S_{3, \operatorname{diss}}\left(\bar{U}_{3}-U_{3, \text { eq }}\right),
$$

where $S_{3 \text {,diss }}$ is a mass transfer coefficient and $U_{3, \text { eq }}=U_{3 \text {,eq }}(t)$ is an equilibrium concentration, see [14], [16], [17].

The production rates of $\mathrm{CaCO}_{3}(\mathrm{aq})$ and $\mathrm{H}_{2} \mathrm{O}$ at the carbonation front are modeled by the function

$$
f_{\text {Reac } \Gamma}=\left[\kappa \phi \phi_{\omega}\left(\bar{U}_{1}\right)^{p}\left(\bar{U}_{3}\right)^{q}\right]_{\Gamma(t)}
$$

where

$$
\Gamma(t)=(S(t), t)
$$

and the notation $[f]_{(x, t)}=f(x, t)$ has been used. Parameter $\kappa>0$ is the reaction constant and $p \geq 1, q \geq 1$, denote the partial reaction orders. In addition, the precipitation rate of $\mathrm{CaCO}_{3}(\mathrm{aq})$ is not considered in the model, that is

$$
f_{\text {Prec }}=0 \text {. }
$$

Let $D_{i} i, \in\{1,2,3\}$ represent diffusion coefficients for each species $\bar{U}_{i}$, and 70 let $\phi D_{5}, \phi D_{6}$ stand for transport coefficients of water content in the carbonated and uncarbonated zone, respectively. It is assumed that each $D_{i}, 1 \leq i \leq 6$, $i \neq 4$, is a positive constant $([17])$.

The continuous model proposed in [16, 17] is described by a coupled system of five partial differential equations (PDE) and one ordinary differential 75 equation (ODE) (balance equations); together with the initial, boundary and transmission conditions and the velocity law of the moving front. The five PDEs and the ODE involving the unknown concentrations are presented as follows:

Carbon dioxide mass concentration in water phase, $\bar{U}_{1}=\left[\mathrm{CO}_{2}(a q)\right]$ :

$$
\frac{\partial}{\partial t}\left(\phi \phi_{\omega} \bar{U}_{1}\right)-\frac{\partial}{\partial x}\left(D_{1} \phi \phi_{\omega} \frac{\partial \bar{U}_{1}}{\partial x}\right)=f_{1, \text { Henry }}, \quad 0 \leq x<S(t), \quad 0<t<T .
$$


Carbon dioxide mass concentration in air phase, $\bar{U}_{2}=\left[\mathrm{CO}_{2}(g)\right]$ :

$\frac{\partial}{\partial t}\left(\phi \phi_{a} \bar{U}_{2}\right)-\frac{\partial}{\partial x}\left(D_{2} \phi \phi_{a} \frac{\partial \bar{U}_{2}}{\partial x}\right)=f_{2, \text { Henry }}, \quad 0 \leq x<S(t), \quad 0<t<T$.

Calcium hydroxide present in the cement, $\bar{U}_{3}=\left[\mathrm{Ca}(\mathrm{OH})_{2}(a q)\right]$ :

$\frac{\partial}{\partial t}\left(\phi \phi_{\omega} \bar{U}_{3}\right)-\frac{\partial}{\partial x}\left(D_{3} \phi \phi_{\omega} \frac{\partial \bar{U}_{3}}{\partial x}\right)=f_{\text {Diss }}, \quad S(t)<x \leq L, \quad 0<t<T$.

The calcium carbonate creation in the carbonation front $\bar{U}_{4}=\left[\mathrm{CaCO}_{3}(a q)\right]$ is given by

$$
\left[\frac{d}{d t}\left(\phi \phi_{\omega} \bar{U}_{4}\right)\right]_{\Gamma(t)}=f_{\text {Prec }}+f_{\text {Reac } \Gamma}, \quad 0<t<T .
$$

Water content in the carbonated zone, $\bar{U}_{5}=\left[\mathrm{H}_{2} \mathrm{O}\right]$,

$$
\frac{\partial}{\partial t}\left(\phi \bar{U}_{5}\right)-\frac{\partial}{\partial x}\left(D_{5} \phi \frac{\partial \bar{U}_{5}}{\partial x}\right)=0,0 \leq x<S(t), 0<t<T .
$$

The last balance equation for water content in the uncarbonated zone, $\bar{U}_{6}=$ $\left[\mathrm{H}_{2} \mathrm{O}\right]$, is given by

$$
\frac{\partial}{\partial t}\left(\phi \bar{U}_{6}\right)-\frac{\partial}{\partial x}\left(D_{6} \phi \frac{\partial \bar{U}_{6}}{\partial x}\right)=0, \quad S(t)<x \leq L, \quad 0<t<T .
$$

Initial position of the front is $S(0)=S_{0}>0$ and initial concentrations in their respective domains take the form

$$
\begin{gathered}
\bar{U}_{i}(x, 0)=\bar{U}_{i 0}(x), \quad 0<x<S_{0}, \quad i \in\{1,2,5\}, \\
\bar{U}_{4}(0)=\bar{U}_{40}, \\
\bar{U}_{i}(x, 0)=\bar{U}_{i 0}(x), \quad S_{0}<x<L, \quad i \in\{3,6\} .
\end{gathered}
$$


With respect to the boundary conditions at the exposed boundary $x=0$ and the sealed boundary $x=L$, one gets

$$
\begin{gathered}
\phi \phi_{\omega} \bar{U}_{1}(0, t)=\Lambda_{1}(t), \phi \phi_{a} \bar{U}_{2}(0, t)=\Lambda_{2}(t), \phi \bar{U}_{5}(0, t)=\Lambda_{5}(t), 0<t<T, \\
\frac{\partial \bar{U}_{3}}{\partial x}(L, t)=0, \quad \frac{\partial \bar{U}_{6}}{\partial x}(L, t)=0,0<t<T .
\end{gathered}
$$
given by

$$
\begin{gathered}
\left(-\delta_{1 i}+\delta_{3 i}\right)\left[\frac{\partial}{\partial x}\left(D_{i} \phi \phi_{\omega} \bar{U}_{i}\right)\right]_{\Gamma(t)}=-\eta_{\Gamma}\left(\bar{U}_{1}, \bar{U}_{3}\right)+S^{\prime}(t)\left[\phi \phi_{\omega} \bar{U}_{i}\right]_{\Gamma(t)}, \quad i \in\{1,3\} \\
-\left[\frac{\partial}{\partial x}\left(D_{2} \phi \phi_{a} \bar{U}_{2}\right)\right]_{\Gamma(t)}=S^{\prime}(t)\left[\phi \phi_{a} \bar{U}_{2}\right]_{\Gamma(t)}, \\
\left(-\delta_{5 i}+\delta_{6 i}\right)\left[\frac{\partial}{\partial x}\left(D_{i} \phi \bar{U}_{i}\right)\right]_{\Gamma(t)}=\delta_{5 i} \frac{1}{\phi_{\omega}} \eta_{\Gamma}\left(\bar{U}_{1}, \bar{U}_{3}\right)+S^{\prime}(t)\left[\phi \bar{U}_{i}\right]_{\Gamma(t)}, \quad i \in\{5,6\} .
\end{gathered}
$$

Finally, the velocity law for propagation of the carbonation front takes the form

$$
S^{\prime}(t)=\left[\alpha \kappa\left(\bar{U}_{1}\right)^{p}\left(\bar{U}_{3}\right)^{q-1}\right]_{\Gamma(t)},
$$

where $\alpha>1$ (16], pp. 544), and the function $\eta_{\Gamma}$ is defined for a couple of functions $V_{1}(x, t)$ and $V_{3}(x, t)$ as

$$
\eta_{\Gamma}\left(V_{1}, V_{3}\right)=\kappa \phi \phi_{\omega}\left(V_{1}(S(t), t)\right)^{p}\left(V_{3}(S(t), t)\right)^{q}, \quad 0<t<T,
$$

so that $\eta_{\Gamma}\left(\bar{U}_{i}, \bar{U}_{i}\right)=f_{\text {Reac }}$, see (5).

As the active concentrations are small, the constant porosity assumption is 
valid (see [2, 13, 22]), thus in this paper the parameters porosity $\phi>0$ as well as water and air fractions $\phi_{\omega}>0$ and $\phi_{a}>0$ are constants. Let us consider the suitable transformation of variables:

$$
\begin{gathered}
\hat{U}_{i}(x, t)=\phi \phi_{\omega} \bar{U}_{i}(x, t), \quad i \in\{1,3\}, \\
\hat{U}_{2}(x, t)=\phi \phi_{a} \bar{U}_{2}(x, t), \quad \hat{U}_{4}(t)=\phi \phi_{\omega} \bar{U}_{4}(t), \\
\hat{U}_{i}(x, t)=\phi \bar{U}_{i}(x, t), \quad i \in\{5,6\} .
\end{gathered}
$$

Under the new variables $\hat{U}_{i}(x, t), i \in\{1,2,3,5,6\}, \hat{U}_{4}(t)$ and using Kronecker's symbol $\delta_{i j}$, problem (8)-(22) can be written in a compact form

$$
\begin{gathered}
\left(\delta_{1 i}+\delta_{2 i}+\delta_{5 i}\right) \frac{\partial \hat{U}_{i}}{\partial t}-D_{i} \frac{\partial^{2} \hat{U}_{i}}{\partial x^{2}}=\left(1-\delta_{5 i}\right)(-1)^{i} P_{i}\left(\hat{U}_{1}-Q_{i} \hat{U}_{2}\right) \\
0 \leq x<S(t), \quad 0<t<T, \quad i \in\{1,2,5\} \\
\frac{\partial \hat{U}_{i}}{\partial t}-D_{i} \frac{\partial^{2} \hat{U}_{i}}{\partial x^{2}}=\delta_{3 i}\left(-S_{3, \text { diss }}\left(\hat{U}_{3}-U_{3, \mathrm{eq}}\right)\right), \quad S(t)<x \leq L, \quad 0<t<T, \quad i \in\{3,6\} \\
\frac{d \hat{U}_{4}}{d t}=\kappa\left(\phi \phi_{\omega}\right)^{1-p-q}\left[\left(\hat{U}_{1}\right)^{p}\left(\hat{U}_{3}\right)^{q}\right]_{\Gamma(t)}, \quad 0<t<T .
\end{gathered}
$$

The transformed initial conditions become $S(0)=S_{0}>0$ and

$$
\begin{gathered}
\hat{U}_{1}(x, 0)=\hat{U}_{10}(x)=\phi \phi_{\omega} \bar{U}_{10}(x), \hat{U}_{2}(x, 0)=\hat{U}_{20}(x)=\phi \phi_{a} \bar{U}_{20}(x), \\
\hat{U}_{5}(x, 0)=\hat{U}_{50}(x)=\phi \bar{U}_{50}(x), 0<x<S_{0} \\
\hat{U}_{4}(0)=\hat{U}_{40}=\phi \phi_{\omega} \bar{U}_{40}
\end{gathered}
$$




$$
\hat{U}_{3}(x, 0)=\hat{U}_{30}(x)=\phi \phi_{\omega} \bar{U}_{30}(x), \quad \hat{U}_{6}(x, 0)=\hat{U}_{60}(x)=\phi \bar{U}_{60}(x), \quad S_{0}<x<L,
$$

and the transformed boundary conditions are

$$
\begin{gathered}
\hat{U}_{i}(0, t)=\Lambda_{i}(t), \quad 0<t<T, \quad i \in\{1,2,5\}, \\
\frac{\partial \hat{U}_{i}}{\partial x}(L, t)=0, \quad 0<t<T, \quad i \in\{3,6\} .
\end{gathered}
$$

Finally, the transformed interface conditions for $x=S(t), 0<t<T$, become

$$
-\left[D_{i} \frac{\partial \hat{U}_{i}}{\partial x}\right]_{\Gamma(t)}=\left(\delta_{5 i}-\delta_{1 i}\right)\left(\phi \phi_{\omega}\right)^{-p-q} \eta_{\Gamma}\left(\hat{U}_{1}, \hat{U}_{3}\right)+S^{\prime}(t)\left[\hat{U}_{i}\right]_{\Gamma(t)}, \quad i \in\{1,2,5\}
$$

$$
\left[D_{i} \frac{\partial \hat{U}_{i}}{\partial x}\right]_{\Gamma(t)}=-\left(\delta_{3 i}\right)\left(\phi \phi_{\omega}\right)^{-p-q} \eta_{\Gamma}\left(\hat{U}_{1}, \hat{U}_{3}\right)+S^{\prime}(t)\left[\hat{U}_{i}\right]_{\Gamma(t)}, \quad i \in\{3,6\}
$$

and the velocity law is

$$
S^{\prime}(t)=\alpha \kappa\left(\phi \phi_{\omega}\right)^{1-p-q}\left[\left(\hat{U}_{1}\right)^{p}\left(\hat{U}_{3}\right)^{q-1}\right]_{\Gamma(t)} .
$$

In this paper, a finite difference method to solve the moving boundary problem (8)-(22) is proposed, obtaining sufficient step sizes conditions to guarantee positivity and stability of the numerical solution. The paper is organized as follows. In Section 2, after a front-fixing transformation, the original problem is transformed into another one in a fixed domain, where the moving boundary becomes a new unknown of the problem. In Section 3, we propose a coupled finite difference scheme whose unknowns are $\left[\mathrm{CO}_{2}(\mathrm{aq})\right],\left[\mathrm{CO}_{2}(\mathrm{~g})\right],\left[\mathrm{CaCO}_{3}(\mathrm{aq})\right]$ and $\left[\mathrm{H}_{2} \mathrm{O}\right]$ in the carbonated zone, $\left[\mathrm{Ca}(\mathrm{OH})_{2}(\mathrm{aq})\right]$ and $\left[\mathrm{H}_{2} \mathrm{O}\right]$ in the uncarbonated 
95 zone, together with the square power values of the expanding front. In Section 4, positivity, boundedness and stability of the numerical solution is treated. Spatial monotonicity properties of the solution is addressed in Section 5. Experiments illustrating the qualitative properties of the numerical solution are included in the corresponding sections.

\section{Front-fixing transformation}

For the sake of simplicity, and taking advance of the fact that $\left(S^{2}(t)\right)^{\prime}=$ $2 S(t) S^{\prime}(t)$, in the following we will consider as unknown the square of the free boundary $R(t)$ instead of the free boundary itself $S(t)$ in order to obtain a more simplified PDE system, i. e.

$$
R(t)=S^{2}(t)
$$

In order to transform the PDE problem with moving domain into a fixed domain one, let us consider the following change of spatial variable inspired by the well known Landau transformation, $([4,12])$ :

$$
z(x, t)= \begin{cases}(x / \sqrt{R(t)})-1, & 0 \leq x<\sqrt{R(t)}, \quad 0 \leq t \leq T, \\ 0, & x=\sqrt{R(t)}, \quad 0 \leq t \leq T, \\ (x-\sqrt{R(t)}) /(L-\sqrt{R(t)}), & \sqrt{R(t)}<x \leq L, \quad 0 \leq t \leq T .\end{cases}
$$

The new unknown variables of the problem are

$$
\begin{aligned}
& U_{i}(z, t)=\hat{U}_{i}(x, t), \quad i \in\{1,2,5\},-1 \leq z \leq 0, \\
& U_{4}(t)=\hat{U}_{4}(t), \\
& U_{i}(z, t)=\hat{U}_{i}(x, t), \quad i \in\{3,6\}, 0 \leq z \leq 1 .
\end{aligned}
$$

In a similar way as in (6), let us denote

$$
\gamma(t)=(0, t)
$$


Then, the new PDE system in the fixed domain $|z| \leq 1$ is

$$
\begin{aligned}
& \left(\delta_{1 i}+\delta_{2 i}+\delta_{5 i}\right)\left(R(t) \frac{\partial U_{i}}{\partial t}-R^{\prime}(t) \frac{z+1}{2} \frac{\partial U_{i}}{\partial z}\right)-D_{i} \frac{\partial^{2} U_{i}}{\partial z^{2}} \\
& =\left(1-\delta_{5 i}\right)(-1)^{i} P_{i}\left(U_{1}-Q_{i} U_{2}\right) R(t), \quad-1 \leq z<0, \quad 0<t<T, \quad i \in\{1,2,5\}
\end{aligned}
$$

$$
\begin{aligned}
& \left(R(t)+L^{2}-2 L \sqrt{R(t)}\right) \frac{\partial U_{i}}{\partial t}+\left(\frac{R^{\prime}(t)}{2}\left(\frac{L}{\sqrt{R(t)}}-1\right)\right)(z-1) \frac{\partial U_{i}}{\partial z}-D_{i} \frac{\partial^{2} U_{i}}{\partial z^{2}} \\
& =-\delta_{3 i}\left(R(t)+L^{2}-2 L \sqrt{R(t)}\right) S_{3, \text { Diss }}\left(U_{3}-U_{3, \text { eq }}\right), \quad 0<z \leq 1, \quad 0<t<T, \quad i \in\{3,6\}
\end{aligned}
$$

$$
\frac{d U_{4}(t)}{d t}=\kappa\left(\phi \phi_{\omega}\right)^{1-p-q}\left[\left(U_{1}\right)^{p}\left(U_{3}\right)^{q}\right]_{\gamma(t)}, \quad 0<t<T
$$

together with the initial conditions

$$
\begin{gathered}
U_{1}(z, 0)=U_{10}(z)=\phi \phi_{\omega} \bar{U}_{10}\left((z+1) S_{0}\right), \quad U_{2}(z, 0)=U_{20}(z)=\phi \phi_{a} \bar{U}_{20}\left((z+1) S_{0}\right) \\
U_{5}(z, 0)=U_{50}(z)=\phi \bar{U}_{50}\left((z+1) S_{0}\right),-1<z<0 \\
U_{4}(0)=U_{40}=\phi \phi_{\omega} \bar{U}_{40} \\
U_{3}(z, 0)=U_{30}(z)=\phi \phi_{\omega} \bar{U}_{30}\left(z\left(L-S_{0}\right)+S_{0}\right) \\
U_{6}(z, 0)=U_{60}(z)=\phi \bar{U}_{60}\left(z\left(L-S_{0}\right)+S_{0}\right), \quad 0<z<1
\end{gathered}
$$

the boundary conditions

$$
\begin{gathered}
U_{i}(-1, t)=\Lambda_{i}(t), \quad 0<t<T, \quad i \in\{1,2,5\}, \\
\frac{\partial U_{i}}{\partial z}(1, t)=0, \quad 0<t<T, \quad i \in\{3,6\},
\end{gathered}
$$


and the interface conditions at $z=0,0<t<T$,

$$
-\left[D_{i} \frac{\partial U_{i}}{\partial z}\right]_{\gamma(t)}=\left(\delta_{5 i}-\delta_{1 i}\right)\left(\phi \phi_{\omega}\right)^{-p-q} \eta_{\gamma}\left(U_{1}, U_{3}\right) \sqrt{R(t)}+\frac{1}{2} R^{\prime}(t)\left[U_{i}\right]_{\gamma(t)}, \quad i \in\{1,2,5\}
$$

$$
\begin{gathered}
{\left[D_{i} \frac{\partial U_{i}}{\partial z}\right]_{\gamma(t)}=-\left(\delta_{3 i}\right)\left(\phi \phi_{\omega}\right)^{-p-q} \eta_{\gamma}\left(U_{1}, U_{3}\right)(L-\sqrt{R(t)})} \\
+\left(\frac{R^{\prime}(t)}{2}\left(\frac{L}{\sqrt{R(t)}}-1\right)\right)\left[U_{i}\right]_{\gamma(t)}, \quad i \in\{3,6\} \\
R^{\prime}(t)=2 \alpha \kappa\left(\phi \phi_{\omega}\right)^{1-p-q} \sqrt{R(t)}\left[\left(U_{1}\right)^{p}\left(U_{3}\right)^{q-1}\right]_{\gamma(t)},
\end{gathered}
$$

where the function $\eta_{\gamma}$ is defined for a couple of functions $V_{1}(z, t)$ and $V_{3}(z, t)$ analogously to (23)

$$
\eta_{\gamma}\left(V_{1}, V_{3}\right)=\kappa \phi \phi_{\omega}\left(V_{1}(0, t)\right)^{p}\left(V_{3}(0, t)\right)^{q}, \quad 0<t<T .
$$

From (51) for $i=3$ and (52), one gets

$$
\left[D_{3} \frac{\partial U_{3}}{\partial z}\right]_{\gamma(t)}=(\alpha-1) \kappa\left(\phi \phi_{\omega}\right)^{1-p-q}\left[\left(U_{1}\right)^{p}\left(U_{3}\right)^{q}\right]_{\gamma(t)}(L-\sqrt{R(t)}) .
$$

Equation (54) shows that $\left[\partial U_{3} / \partial z\right]_{\gamma(t)}$ is positive. This fact will be used in Section 3 dealing with the numerical solution.

\section{Discretization and numerical scheme construction}

In this Section we construct a finite difference scheme for solving numerically the coupled system (42)-(52). Let $M$ and $N$ be positive integers, so that the domain $[-1,1] \times[0, T]$ is partitioned in $(2 M+1) \times(N+1)$ mesh points denoted by 
${ }_{110}\left(z_{j}, t^{n}\right)$, where $z_{j}=j h,-M \leq j \leq M$ and $t^{n}=n k, 0 \leq n \leq N$. Here the step sizes discretizations $h$ and $k$ verify $h M=1$ and $k N=T$, respectively. Numerical approximations of the involved variables are denoted by $u_{i, j}^{n} \approx U_{i}\left(z_{j}, t^{n}\right), i \in$ $\{1,2,3,5,6\}, u_{4}^{n} \approx U_{4}\left(t^{n}\right), r^{n} \approx R\left(t^{n}\right)$, while we denote $\lambda_{i}^{n}=\Lambda_{i}\left(t^{n}\right), i \in$ $\{1,2,5\}$.

115 Partial derivatives at the interior points are approximated using forward in time and centered in space finite difference expressions:

$$
\begin{gathered}
\frac{u_{i, j}^{n+1}-u_{i, j}^{n}}{k} \approx \frac{\partial U_{i}}{\partial t}\left(z_{j}, t^{n}\right), \quad, \frac{u_{i, j+1}^{n}-u_{i, j-1}^{n}}{2 h} \approx \frac{\partial U_{i}}{\partial z}\left(z_{j}, t^{n}\right), \\
\frac{u_{i, j+1}^{n}-2 u_{i, j}^{n}+u_{i, j-1}^{n}}{h^{2}} \approx \frac{\partial^{2} U_{i}}{\partial z^{2}}\left(z_{j}, t^{n}\right), \quad i \in\{1,2,3,5,6\} \\
\frac{u_{4}^{n+1}-u_{4}^{n}}{k} \approx \frac{d U_{4}}{d t}\left(t^{n}\right), \frac{r^{n+1}-r^{n}}{k} \approx R^{\prime}\left(t^{n}\right) .
\end{gathered}
$$

With respect to the discretization of the first derivatives of the transformed transmission conditions at the carbonation front $z=0$, we use one side second order finite difference approximations. We take left hand side approximations with three points for the discretization at the carbonated zone:

$$
\frac{3 u_{i, 0}^{n}-4 u_{i,-1}^{n}+u_{i,-2}^{n}}{2 h} \approx \frac{\partial U_{i}}{\partial z}\left(0, t^{n}\right), \quad i \in\{1,2,5\}
$$

and the right side approximations for the discretization at the uncarbonated zone

$$
\frac{-3 u_{i, 0}^{n}+4 u_{i, 1}^{n}-u_{i, 2}^{n}}{2 h} \approx \frac{\partial U_{i}}{\partial z}\left(0, t^{n}\right), \quad i \in\{3,6\} .
$$

Using the approximations (55)-(58), equations (42)-(44) become discretized 
at the interior mesh points in the following way

$$
\begin{aligned}
& \left(\delta_{1 i}+\delta_{2 i}+\delta_{5 i}\right) r^{n} \frac{u_{i, j}^{n+1}-u_{i, j}^{n}}{k}-\frac{1+z_{j}}{2}\left(\frac{r^{n+1}-r^{n}}{k}\right) \frac{u_{i, j+1}^{n}-u_{i, j-1}^{n}}{2 h} \\
& -D_{i} \frac{u_{i, j+1}^{n}-2 u_{i, j}^{n}+u_{i, j-1}^{n}}{h^{2}}=\left(1-\delta_{5 i}\right)(-1)^{i} r^{n} P_{i}\left(u_{1, j}^{n}-Q_{i} u_{2, j}^{n}\right), \\
& -M+1 \leq j \leq-1, \quad 0 \leq n \leq N-1, \quad i \in\{1,2,5\}, \\
& \left(r^{n}+L^{2}-2 L \sqrt{r^{n}}\right) \frac{u_{i, j}^{n+1}-u_{i, j}^{n}}{k}+\frac{z_{j}-1}{2}\left(\frac{r^{n+1}-r^{n}}{k}\right)\left(\frac{L}{\sqrt{r^{n}}}-1\right) \frac{u_{i, j+1}^{n}-u_{i, j-1}^{n}}{2 h} \\
& -D_{i} \frac{u_{i, j+1}^{n}-2 u_{i, j}^{n}+u_{i, j-1}^{n}}{h^{2}}-\delta_{3 i}\left(r^{n}+L^{2}-2 L \sqrt{r^{n}}\right) S_{3, \mathrm{diss}}\left(u_{3, j}^{n}-u_{3, \mathrm{eq}}^{n}\right), \\
& 1 \leq j \leq M, \quad 0 \leq n \leq N-1, \quad i \in\{3,6\} .
\end{aligned}
$$

In equation (60), for $j=M$, it appears the concentrations $u_{i, M+1}^{n}, i \in\{3,6\}$, corresponding to the mesh point $z_{M+1}=(M+1) h$ out of the numerical domain. To find the value of $u_{i, M+1}^{n}$, the finite difference approximations of the boundary conditions at the sealed surface are used as follows, see (49),

$$
\frac{u_{i, M+1}^{n}-u_{i, M-1}^{n}}{2 h}=0, \quad 0 \leq n \leq N, \quad i \in\{3,6\},
$$

obtaining $u_{i, M+1}^{n}=u_{i, M-1}^{n}$.

The discretization of the ODE (44) takes the form

$$
\frac{u_{4}^{n+1}-u_{4}^{n}}{k}=\kappa\left(\phi \phi_{\omega}\right)^{1-p-q}\left(u_{1,0}^{n}\right)^{p}\left(u_{3,0}^{n}\right)^{q}, \quad 0 \leq n \leq N-1 .
$$

Initial concentrations given in (45)-(47) take the discrete form

$$
\begin{aligned}
& u_{1, j}^{0}=U_{10}\left(z_{j}\right)=\phi \phi_{\omega} \bar{U}_{10}\left(z_{j} S_{0}\right), \quad u_{2, j}^{0}=U_{20}\left(z_{j}\right)=\phi \phi_{a} \bar{U}_{20}\left(z_{j} S_{0}\right), \\
& u_{5, j}^{0}=U_{50}\left(z_{j}\right)=\phi \bar{U}_{50}\left(z_{j} S_{0}\right), \quad-M+1 \leq j \leq-1,
\end{aligned}
$$

$$
u_{4}^{0}=U_{40}=\phi \phi_{\omega} \bar{U}_{40},
$$


$u_{3, j}^{0}=U_{30}\left(z_{j}\right)=\phi \phi_{\omega} \bar{U}_{30}\left(z_{j} S_{0}\right), \quad u_{6, j}^{0}=U_{60}\left(z_{j}\right)=\phi \bar{U}_{60}\left(z_{j} S_{0}\right), \quad 1 \leq j \leq M$.

Boundary conditions at the exposed surface take the values

$$
u_{i,-M}^{n}=\lambda_{i}^{n}, \quad 0 \leq n \leq N, \quad i \in\{1,2,5\} .
$$

The discretization of the Stefan-like conditions (50)-(52) takes the form

$$
\begin{aligned}
& -D_{i} \frac{3 u_{i, 0}^{n}-4 u_{i,-1}^{n}+u_{i,-2}^{n}}{2 h}=\left(\delta_{5 i}-\delta_{1 i}\right) \kappa\left(\phi \phi_{\omega}\right)^{1-p-q} \sqrt{r^{n}}\left(u_{1,0}^{n}\right)^{p}\left(u_{3,0}^{n}\right)^{q} \\
& +\frac{1}{2} \frac{r^{n+1}-r^{n}}{k} u_{i, 0}^{n}, \quad 0 \leq n \leq N-1, \quad i \in\{1,2,5\}, \\
& -D_{i} \frac{3 u_{i, 0}^{n}-4 u_{i, 1}^{n}+u_{i, 2}^{n}}{2 h}=-\delta_{3 i} \kappa\left(\phi \phi_{\omega}\right)^{1-p-q}\left(L-\sqrt{r^{n}}\right)\left(u_{1,0}^{n}\right)^{p}\left(u_{3,0}^{n}\right)^{q} \\
& +\frac{1}{2} \frac{r^{n+1}-r^{n}}{k}\left(\frac{L}{\sqrt{r^{n}}}-1\right) u_{i, 0}^{n}, \quad 0 \leq n \leq N-1, \quad i \in\{3,6\},
\end{aligned}
$$

and the discretization of the propagation velocity of the carbonation front

$$
\frac{r^{n+1}-r^{n}}{k}=2 \alpha \kappa\left(\phi \phi_{\omega}\right)^{1-p-q} \sqrt{r^{n}}\left(u_{1,0}^{n}\right)^{p}\left(u_{3,0}^{n}\right)^{q-1}, 0 \leq n \leq N-1 .
$$

Equations (59) and (60) provide the numerical solution at the interior points. The values at the natural boundaries (exposed $z=-1$ and sealed $z=1$ ) are given by (66) and (61), respectively.

With respect to the unknown concentrations at the interface boundary $z=$ 0 at each temporal step $n, u_{i, 0}^{n}, i \in\{1,2,3,5,6\}$, the driving equations take the form (67), (68) and (69), building a nonlinear system involving also the discretized transformed interface boundary $r^{n+1}$. Note that, from (67) and (68), the unknown $u_{i, 0}^{n}, 1 \leq i \leq 6, i \neq 4$ depend on three unknown $r^{n+1}, u_{1,0}^{n}$ and $u_{3,0}^{n}$, i. e. $u_{i, 0}^{n}=\xi_{i}\left(r^{n+1}, u_{1,0}^{n}, u_{3,0}^{n}\right)$. Also, from (69),$r^{n+1}$ depends on $u_{1,0}^{n}$ and $u_{3,0}^{n}$. Thus, $r^{n+1}=\psi\left(u_{1,0}^{n}, u_{3,0}^{n}\right)$. Hence, let us consider the subsystem of three equations (67) fixing $i=1$, (68) fixing $i=3$ and (69), whose unknows 
are $u_{1,0}^{n}, u_{3,0}^{n}$ and $r^{n+1}$. After straightforward manipulations of these equations, one gets firstly $u_{3,0}^{n}$ from

$$
\begin{aligned}
& h^{\frac{1}{p}} \sqrt{r^{n}}\left(\left(L-\sqrt{r^{n}}\right)(\alpha-1)\right)^{\frac{1}{p}} \frac{D_{3}}{2}\left(-3 u_{3,0}^{n}+4 u_{3,1}^{n}-u_{3,2}^{n}\right)\left(u_{3,0}^{n}\right)^{\frac{p+q}{p}} \\
& +h^{\frac{1}{p}}\left(\left(L-\sqrt{r^{n}}\right)(\alpha-1)\right)^{\frac{p+1}{p}} \frac{D_{1}}{2}\left(4 u_{1,-1}^{n}-u_{1,-2}^{n}\right)\left(u_{3,0}^{n}\right)^{\frac{p+q}{p}} \\
& -\left(L-\sqrt{r^{n}}\right)(\alpha-1) \kappa^{-\frac{1}{p}}\left(\phi \phi_{\omega}\right)^{\frac{p+q-1}{p}} \frac{3 D_{1}}{2}\left(\frac{D_{3}}{2}\left(-3 u_{3,0}^{n}+4 u_{3,1}^{n}-u_{3,2}^{n}\right)\right)^{\frac{1}{p}} u_{3,0}^{n} \\
& -\sqrt{r^{n}} \alpha \kappa^{-\frac{1}{p}}\left(\phi \phi_{\omega}\right)^{\frac{p+q-1}{p}}\left(\frac{D_{3}}{2}\left(-3 u_{3,0}^{n}+4 u_{3,1}^{n}-u_{3,2}^{n}\right)\right)^{\frac{p+1}{p}}=0, \quad 0 \leq n \leq N-1 .
\end{aligned}
$$

Note that expression (70) is well defined when $u_{3,0}^{n}$ is positive and the basis of the powers arising in (70) are also positive. Since $\alpha>1$ and the carbonation front $\sqrt{r^{n}}$ is on the left of the sealed boundary $z=1$, one gets that coefficient $\left(L-\sqrt{r^{n}}\right)(\alpha-1)$ is positive. Otherwise, from (58) for $i=3$, and positivity of the partial derivative $\left[\partial U_{3} / \partial z\right]_{\gamma(t)}$ in (54), it holds that $\left(-3 u_{3,0}^{n}+4 u_{3,1}^{n}-u_{3,2}^{n}\right)$

${ }_{130}$ is positive for small enough values of $h$.

The discussion of existence and uniqueness of solution of (70) will be treated at the end of this section.

Once the value $u_{3,0}^{n}$ is found, $u_{1,0}^{n}$ is calculated using (67) for $i=1$ and (69) by

$u_{1,0}^{n}=\left\{D_{3} \frac{-3 u_{3,0}^{n}+4 u_{3,1}^{n}-u_{3,2}^{n}}{2 h}\left(\kappa\left(\phi \phi_{\omega}\right)^{1-p-q}\left(u_{3,0}^{n}\right)^{q}\left(L-\sqrt{r^{n}}\right)(\alpha-1)\right)^{-1}\right\}^{\frac{1}{p}}$,

$0 \leq n \leq N-1$

and for the discretized free boundary value $r^{n+1}$ one gets

$$
r^{n+1}=r^{n}+2 k \alpha \kappa\left(\phi \phi_{\omega}\right)^{1-p-q} \sqrt{r^{n}}\left(u_{1,0}^{n}\right)^{p}\left(u_{3,0}^{n}\right)^{q-1}, \quad 0 \leq n \leq N-1 .
$$


Note that carbonation front $r^{n}$ is time increasing while $u_{3,0}^{n}$ be positive, because in such situation $u_{1,0}^{n}$ will be also positive.

Then, values $u_{1,0}^{n}, u_{3,0}^{n}$ and $r^{n+1}$ are obtained, the solution of system (67)(68) provides the values of the remaining unknowns $u_{i, 0}^{n}, i=2,5,6$. From (67), for $i=2$

$$
u_{2,0}^{n}=\frac{D_{2}}{2 h}\left(4 u_{2,-1}^{n}-u_{2,-2}^{n}\right)\left(\frac{1}{2} \frac{r^{n+1}-r^{n}}{k}+\frac{3 D_{2}}{2 h}\right)^{-1}, 0 \leq n \leq N-1 .
$$

From (67), for $i=5$

$$
\begin{aligned}
& u_{5,0}^{n}=\left(\frac{D_{5}}{2 h}\left(4 u_{5,-1}^{n}-u_{5,-2}^{n}\right)-\kappa\left(\phi \phi_{\omega}\right)^{1-p-q} \sqrt{r^{n}}\left(u_{1,0}^{n}\right)^{p}\left(u_{3,0}^{n}\right)^{q}\right)\left(\frac{1}{2} \frac{r^{n+1}-r^{n}}{k}+\frac{3 D_{5}}{2 h}\right)^{-1}, \\
& 0 \leq n \leq N-1 .
\end{aligned}
$$

And finally, from (68), for $i=6$

$$
u_{6,0}^{n}=\frac{D_{6}}{2 h}\left(4 u_{6,1}^{n}-u_{6,2}^{n}\right)\left(\frac{1}{2} \frac{r^{n+1}-r^{n}}{k}\left(\frac{L}{\sqrt{r^{n}}}-1\right)+\frac{3 D_{6}}{2 h}\right)^{-1}, 0 \leq n \leq N-1 .
$$

From equations (59)-(60) the solutions at the interior points at time level $n+1$ are given by:

$$
\begin{aligned}
& u_{i, j}^{n+1}=a_{i, j}^{n} u_{i, j-1}^{n}+b_{i, j}^{n} u_{i, j}^{n}+c_{i, j}^{n} u_{i, j+1}^{n}+\delta_{1 i} k P_{1} Q_{1} u_{2, j}^{n}+\delta_{2 i} k P_{2} u_{1, j}^{n}, \\
& -M+1 \leq j \leq-1, \quad 0 \leq n \leq N-1, \quad i \in\{1,2,5\} \\
& u_{i, j}^{n+1}=a_{i, j}^{n} u_{i, j-1}^{n}+b_{i, j}^{n} u_{i, j}^{n}+c_{i, j}^{n} u_{i, j+1}^{n}+\delta_{3 i} k S_{3, \mathrm{diss}} u_{3, \mathrm{eq}}^{n}, \\
& 1 \leq j \leq M, \quad 0 \leq n \leq N-1, \quad i \in\{3,6\}
\end{aligned}
$$

where

$$
\begin{gathered}
a_{i, j}^{n}= \begin{cases}\frac{D_{i} k}{h^{2} r^{n}}-\frac{1+z_{j}}{4 h} \Delta_{1}^{n}, & i \in\{1,2,5\}, \\
\frac{D_{i} k}{h^{2} \Delta_{3}^{n}}+\frac{z_{j}-1}{4 h} r^{n} \frac{\Delta_{1}^{n} \Delta_{2}^{n}}{\Delta_{3}^{n}}, & i \in\{3,6\},\end{cases} \\
b_{i, j}^{n}= \begin{cases}1-\frac{2 D_{i} k}{h^{2} r^{n}}-\delta_{1 i} k P_{1}-\delta_{2 i} k P_{2} Q_{2}, & i \in\{1,2,5\}, \\
1-\frac{2 D_{i} k}{h^{2} \Delta_{3}^{n}}-\delta_{3 i} k S_{3, \text { diss }}, & i \in\{3,6\},\end{cases}
\end{gathered}
$$




$$
c_{i, j}^{n}= \begin{cases}\frac{D_{i} k}{h^{2} r^{n}}+\frac{1+z_{j}}{4 h} \Delta_{1}^{n}, & i \in\{1,2,5\}, \\ \frac{D_{i} k}{h^{2} \Delta_{3}^{n}}+\frac{1-z_{j}}{4 h} r^{n} \frac{\Delta_{1}^{n} \Delta_{2}^{n}}{\Delta_{3}^{n}}, & i \in\{3,6\},\end{cases}
$$

and

$$
\Delta_{1}^{n}=\frac{r^{n+1}}{r^{n}}-1, \quad \Delta_{2}^{n}=\frac{L}{\sqrt{r^{n}}}-1, \quad \Delta_{3}^{n}=\left(L-\sqrt{r^{n}}\right)^{2}, 0 \leq n \leq N-1 .
$$

Finally, from (62), the concentration of $\mathrm{CaCO}_{3}(\mathrm{aq})$ in the carbonation front at the step $n+1$ is given by

$$
u_{4}^{n+1}=u_{4}^{n}+k \kappa\left(\phi \phi_{\omega}\right)^{1-p-q}\left(u_{1,0}^{n}\right)^{p}\left(u_{3,0}^{n}\right)^{q}, \quad 0 \leq n \leq N-1 .
$$

We conclude this Section with the solvability for $u_{3,0}^{n}$ of the nonlinear equation (700). Note that this equation (70) can be rewritten in the compact form $F_{n}(\xi)=A_{n}\left(B_{n}-3 \xi\right) \xi^{\frac{p+q}{p}}+C_{n} \xi^{\frac{p+q}{p}}-D_{n}\left(B_{n}-3 \xi\right)^{\frac{1}{p}} \xi-E_{n}\left(B_{n}-3 \xi\right)^{\frac{p+1}{p}}=0$.

Here, coefficients of equation (83) are given by

$$
\begin{aligned}
& K_{1, n}=\left(L-\sqrt{r^{n}}\right)(\alpha-1), \quad K_{2, n}=4 u_{3,1}^{n}-u_{3,2}^{n}, \quad K_{3, n}=4 u_{1,-1}^{n}-u_{1,-2}^{n}, \\
& A_{n}=h^{\frac{1}{p}} \sqrt{r^{n}}\left(K_{1, n}\right)^{\frac{1}{p}} \frac{D_{3}}{2}, \quad B_{n}=K_{2, n}, \quad C_{n}=h^{\frac{1}{p}}\left(K_{1, n}\right)^{\frac{p+1}{p}} K_{3, n} \frac{D_{1}}{2} \\
& D_{n}=K_{1, n} \kappa^{-\frac{1}{p}}\left(\phi \phi_{\omega}\right)^{\frac{p+q-1}{p}} \frac{3 D_{1}}{2}\left(\frac{D_{3}}{2}\right)^{\frac{1}{p}}, \quad E_{n}=\alpha \sqrt{r^{n}} \kappa^{-\frac{1}{p}}\left(\phi \phi_{\omega}\right)^{\frac{p+q-1}{p}}\left(\frac{D_{3}}{2}\right)^{\frac{p+1}{p}} .
\end{aligned}
$$

From the positivity of the involved coefficients of the expression of $E_{n}$ given in (844), it is clear that $E_{n}>0$. Since $\alpha>1$ and the carbonation front $\sqrt{r^{n}}$ is on the left of the sealed boundary $x=L$, one gets that coefficient $K_{1, n}$ is positive. Hence, coefficients $A_{n}$ and $D_{n}$ are also positive. Positivity of coefficients $K_{2, n}$ and $K_{3, n}$ can be proved in a similar way; for the sake of brevity we will state just that $K_{2, n}>0$ for small enough values of $h$. In fact, from the continuous 
spatial differenciability of the theoretical exact solution $U_{3}(z, t)$ ([17], Theorem 3.6) and the mean value theorem, one gets

$$
u_{3,2}^{n}=u_{3,1}^{n}+h \frac{\partial U_{3}}{\partial z}\left(\xi, t^{n}\right) ; \quad h<\xi<2 h
$$

Let $e_{n}$ be defined by

$$
e_{n}=\max \left|\frac{\partial U_{3}}{\partial z}\left(z, t^{n}\right)\right|, \quad 0 \leq z \leq 1,
$$

from (85) and (86)

$$
\left|u_{3,2}^{n}-u_{3,1}^{n}\right|<e_{n} h
$$

Hence, taking $h<3 u_{3,1} / e_{n}$, one gets

$$
K_{2, n}=4 u_{3,1}^{n}-u_{3,2}^{n}=3 u_{3,1}^{n}+\left(u_{3,1}^{n}-u_{3,2}^{n}\right)>3 u_{3,2}^{n}-e_{n} h>0 .
$$

The positivity of $K_{3, n}$ for small enough values of $h$ can be stated in an analogous way.

Hence, the remaining coefficients $B_{n}$ and $C_{n}$ become also positive for small enough values of $h$. The function $F_{n}(\xi)$ given by (83) is well defined, continuous and differentiable as a function of $\xi$ in the interval $0 \leq \xi \leq B_{n} / 3$ and its sign changes in both extremes:

$$
F_{n}(0)=-E_{n}\left(B_{n}\right)^{\frac{p+1}{p}}<0, \quad F_{n}\left(B_{n} / 3\right)=C_{n}\left(B_{n} / 3\right)^{\frac{p+q}{p}}>0
$$

Thus, the equation (83) admits a solution and now we will prove that the solution is unique under appropriate conditions. Taking derivatives in the ex- 
pression of $F_{n}(\xi)$ and using that $0 \leq \xi \leq B_{n} / 3$ one gets

$$
\begin{aligned}
& F_{n}^{\prime}(\xi)=-3 A_{n}\left(\frac{2 p+q}{p}\right) \xi^{\frac{p+q}{p}}+\left(A_{n} B_{n}+C_{n}\right)\left(\frac{p+q}{p}\right) \xi^{\frac{q}{p}} \\
& +\left(3 E_{n}\left(\frac{p+1}{p}\right)-D_{n}\right)\left(B_{n}-3 \xi\right)^{\frac{1}{p}}+\frac{3 D_{n}}{p} \xi\left(B_{n}-3 \xi\right)^{\frac{1-p}{p}} \\
& \geq-3 A_{n}\left(\frac{2 p+q}{p}\right) \frac{B_{n}}{3} \xi^{\frac{q}{p}}+\left(A_{n} B_{n}+C_{n}\right)\left(\frac{p+q}{p}\right) \xi^{\frac{q}{p}} \\
& +\left(3 E_{n}\left(\frac{p+1}{p}\right)-D_{n}\right)\left(B_{n}-3 \xi\right)^{\frac{1}{p}}+\frac{3 D_{n}}{p} \xi\left(B_{n}-3 \xi\right)^{\frac{1-p}{p}} \\
& =\left(-A_{n} B_{n}\left(\frac{2 p+q}{p}\right)+\left(A_{n} B_{n}+C_{n}\right)\left(\frac{p+q}{p}\right)\right) \xi^{\frac{q}{p}} \\
& +\left(3 E_{n}\left(\frac{p+1}{p}\right)-D_{n}\right)\left(B_{n}-3 \xi\right)^{\frac{1}{p}}+\frac{3 D_{n}}{p} \xi\left(B_{n}-3 \xi\right)^{\frac{1-p}{p}}
\end{aligned}
$$

Note that if

$$
\left(A_{n} B_{n}+C_{n}\right)\left(\frac{p+q}{p}\right) \geq A_{n} B_{n}\left(\frac{2 p+q}{p}\right), 3 E_{n}\left(\frac{p+1}{p}\right) \geq D_{n},
$$

then the nonnegativity of the derivative $F_{n}^{\prime}(\xi)$ is guaranteed.

Taking into account (84), note that conditions (91) hold true when

$$
\rho_{1} \leq \frac{\sqrt{r^{n}}}{L} \leq \rho_{2, n}
$$

where

$$
\rho_{1}=1-\frac{\alpha}{(\alpha-1)} \frac{D_{3}}{D_{1}}\left(\frac{p+1}{p}\right), \rho_{2, n}=1-\frac{1}{(\alpha-1)} \frac{D_{3}}{D_{1}}\left(\frac{p}{p+q}\right) \frac{K_{2, n}}{K_{3, n}} .
$$

Note that this means that $u_{3,0}^{n}$ is uniquely determined and positive while the carbonation front $\sqrt{r^{n}}$ satisfies condition (92), i. e., when the carbonation front lies in the set $\left[\rho_{1} L, \rho_{2, n} L\right] \cap[0, L]$.

As we wrote above, see (72), the positivity of $u_{3,0}^{n}$ guarantees that the carbonation front is time increasing, as it is expected.

We can summarize the construction of the numerical solution in the procedure exposed in Algorithm 1 


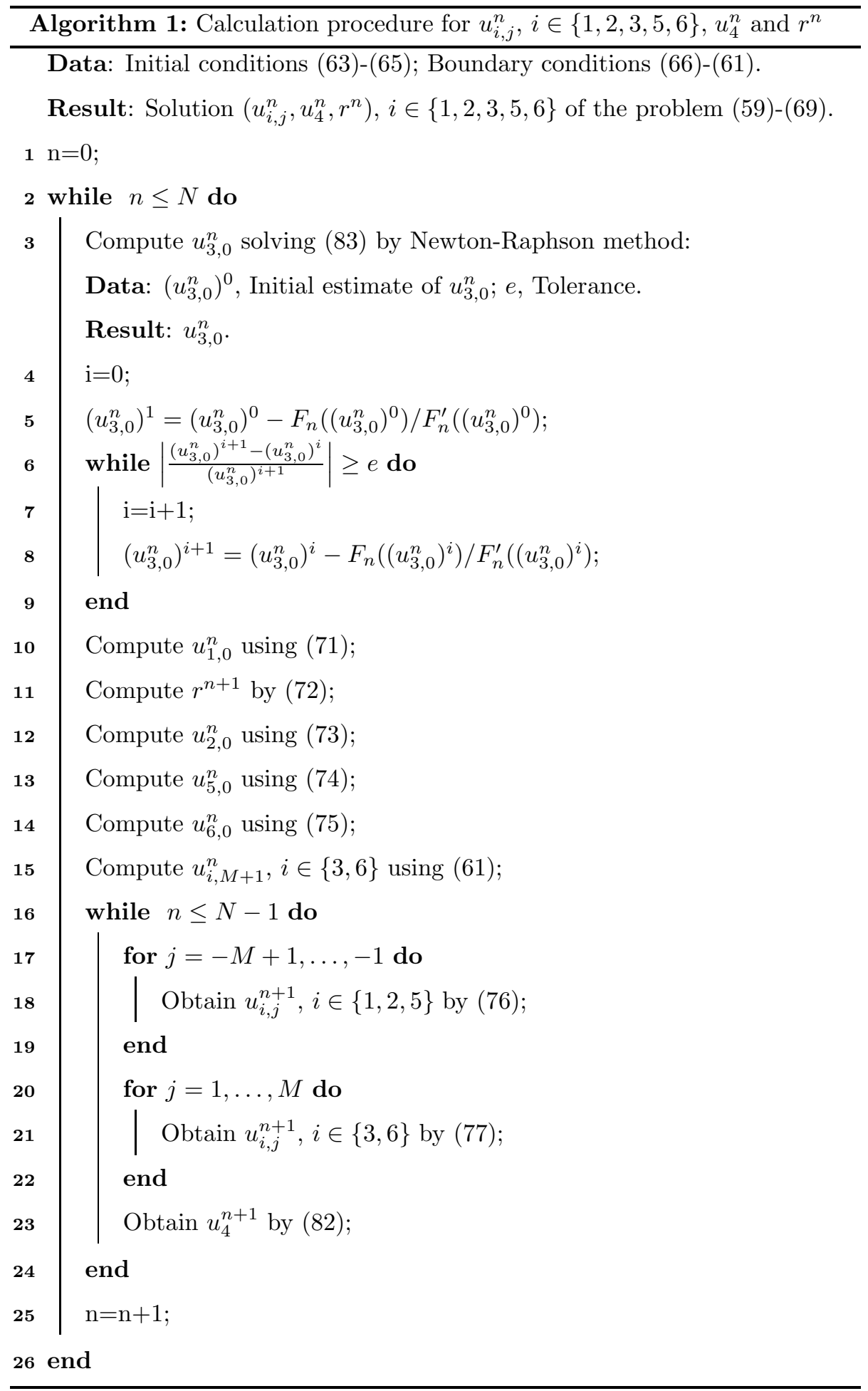




\section{Numerical analysis: stability and positivity}

Dealing with concentrations, the numerical solution of the scheme (76)-(82) together with the values at the boundaries have to be positive. As the best model may be wasted with a disregarded numerical analysis, apart from the positivity it is convenient to study the stability of the numerical solution. Thus,

150 the numerical solution of our scheme will preserve the qualitative properties of the theoretical solution proved in [17], Theorem 3.3.

In this section, we assume the hypothesis of the continuous model about the bounds of initial and boundary concentrations of $\mathrm{CO}_{2}(\mathrm{aq})$ and $\mathrm{CO}_{2}(\mathrm{~g})$ relationships,

$$
Q_{1} \tilde{G}_{2} \leq \tilde{G}_{1}, \quad \tilde{G}_{1} \leq Q_{2} \tilde{G}_{2},
$$

where $\tilde{G}_{1}$ is the upper bound of carbon dioxide mass concentration in water phase, and $\tilde{G}_{2}$ is the upper bound of carbon dioxide mass concentration in air phase, for both at the exposed boundary in any time and in the carbonated zone at the initial time, together with the condition on the equilibrium concentration of calcium hydroxide,

$$
u_{3, \mathrm{eq}}^{n} \leq \tilde{G}_{3},
$$

see [17], Section 3, pp. 240. Here $Q_{1}$ and $Q_{2}$ are introduced in expression (3).

In addition, we will assume the existence of an upper bound $\tilde{G}_{5}$ for the water content for both at the exposed boundary in any time and in the carbonated region at the initial time. Regarding the uncarbonated zone, we will suppose that mass concentration of $\mathrm{Ca}(\mathrm{OH})_{2}(\mathrm{aq})$ and water content are upper-bounded by $\tilde{G}_{3}$ and $\tilde{G}_{6}$, respectively, at the initial time, see [17], Section 3, pp. 239-240.

Let $U_{i 0}\left(z_{j}\right), 1 \leq i \leq 6, i \neq 4$, be the initial conditions given by (63)-(65) and let $\lambda_{i}^{n}, i \in\{1,2,5\}$, be the exposed boundary conditions given by (66). Then, according to the above hypotheses, one can write

$$
\lambda_{i}^{n} \leq \tilde{G}_{i}, \quad i \in\{1,2,5\}, \quad 0 \leq n \leq N,
$$


and

$$
\begin{aligned}
& u_{i, j}^{0}=U_{i 0}\left(z_{j}\right) \leq \tilde{G}_{i}, \quad-M+1 \leq j \leq-1, \quad, i \in\{1,2,5\}, \\
& u_{i, j}^{0}=U_{i 0}\left(z_{j}\right) \leq \tilde{G}_{i}, \quad 1 \leq j \leq M, \quad i \in\{3,6\} .
\end{aligned}
$$

We will find sufficient conditions on the discretization step sizes $h$ and $k$ under which the numerical solution $\left\{u_{i, j}^{n}\right\}$ and $\left\{u_{4}^{n}\right\}$ of the scheme (76)-(82) is positive and bounded. The results are obtained using the induction principle on the temporal index $n$, uniformly on the spatial index $j$.

Firstly, let us derive some numerical results based on the approximation of the spatial partial derivatives of the concentrations at the carbonation front. The right-hand side approximation of the spatial partial derivative of the concentrations of chemical species in the carbonated zone $U_{i}(z, t)$ at $\left(0, t^{n}\right)$ can be written in the form

$$
\frac{-3 u_{i, 0}^{n}+4 u_{i, 1}^{n}-u_{i, 2}^{n}}{2 h}=\frac{\partial U_{i}}{\partial z}\left(0, t^{n}\right)+\mathcal{O}\left(h^{2}\right), \quad i \in\{1,2,5\},
$$

where the artificial values $u_{i, 1}^{n}$ and $u_{i, 2}^{n}$ vanish for $0 \leq n \leq N$ and $i \in\{1,2,5\}$, because they are outside of the real carbonated region.

Otherwise, the left-hand side backward approximation of the spatial partial derivative behaves, according to (57),

$$
\frac{3 u_{i, 0}^{n}-4 u_{i,-1}^{n}+u_{i,-2}^{n}}{2 h}=\frac{\partial U_{i}}{\partial z}\left(0, t^{n}\right)+\mathcal{O}\left(h^{2}\right), \quad i \in\{1,2,5\} .
$$

From (98)-(99), one gets

$$
6 u_{i, 0}^{n}=4 u_{i,-1}^{n}-u_{i,-2}^{n}+\mathcal{O}\left(h^{3}\right), \quad i \in\{1,2,5\} .
$$

On the other hand, let us consider the left-hand side approximation of the spatial partial derivative of $U_{i}(z, t)$ at $\left(0, t^{n}\right)$

$$
\frac{3 u_{i, 0}^{n}-4 u_{i,-1}^{n}+u_{i,-2}^{n}}{2 h}=\frac{\partial U_{i}}{\partial z}\left(0, t^{n}\right)+\mathcal{O}\left(h^{2}\right), \quad i \in\{3,6\},
$$

where the artificial values $u_{i,-1}^{n}$ and $u_{i,-2}^{n}$ vanish. 
The right-hand side backward approximation of the spatial partial derivative is, according to (58)

$$
\frac{-3 u_{i, 0}^{n}+4 u_{i, 1}^{n}-u_{i, 2}^{n}}{2 h}=\frac{\partial U_{i}}{\partial z}\left(0, t^{n}\right)+\mathcal{O}\left(h^{2}\right), \quad i \in\{3,6\}
$$

and from (101)-102), it follows that

$$
6 u_{i, 0}^{n}=4 u_{i, 1}^{n}-u_{i, 2}^{n}+\mathcal{O}\left(h^{3}\right), \quad i \in\{3,6\} .
$$
for both indexes 0 and $n$.

For $n=0$, initial concentrations $u_{i, j}^{0}, 1 \leq i \leq 6, i \neq 4,-M \leq j \leq M, j \neq 0$, and $u_{4}^{0} \geq 0$ are given and non negative. From the results of Section 3, one gets the positivity of $u_{i, 0}^{0}, 1 \leq i \leq 6, i \neq 4$. Using (72) for $n=0$, the transformed carbonation front verifies $r^{1}>r^{0}>0$. Let $\tilde{G}_{i}$ be the positive bounds, such that

$$
0 \leq u_{i, j}^{0} \leq \tilde{G}_{i}, \quad 1 \leq i \leq 6, \quad i \neq 4
$$

Using (100), (103) and (104), one gets

$$
\begin{aligned}
& u_{i, 0}^{0} \leq \frac{2}{3} u_{i,-1}^{0} \leq \tilde{G}_{i}, \quad i \in\{1,2,5\} \\
& u_{i, 0}^{0} \leq \frac{2}{3} u_{i, 1}^{0} \leq \tilde{G}_{i}, \quad i \in\{3,6\}
\end{aligned}
$$

Let us assume the induction hypothesis, i. e., concentrations $u_{i, j}^{n}$ satisfy

$$
0 \leq u_{i, j}^{n} \leq \tilde{G}_{i}, \quad-M \leq j \leq M, \quad 1 \leq i \leq 6, \quad i \neq 4
$$

The behaviour of concentration $u_{4}^{n}$ is different and will be treated later and separately. Note that from (76)-(77), the numerical solution $u_{i, j}^{n+1}$ at the points $-M+1 \leq j \leq M$ is guaranteed to be non negative if coefficients $a_{i, j}^{n}, b_{i, j}^{n}$ and ${ }_{170} c_{i, j}^{n}$ are non negative.

The coefficients $c_{i, j}^{n}$ in equations (76)-(77) and (80) are unconditionally positive. The nonnegativity of the coefficients $a_{i, j}^{n}, i \in\{1,2,5\}$, in equations (76)(77) and (78) will be proved using the value of the difference $r^{n+1}-r^{n}$ that 
appears in the transmission conditions (67). Using the induction principle, positivity of $u_{3,0}^{n}$ and $u_{1,0}^{n}$ and equations (171)-(172), one gets that $r^{n+1}>r^{n}>r^{0}>0$.

Using (67) and (100) for $i=1$, one gets

$$
r^{n+1}-r^{n}=3 D_{1} \frac{k}{h}+2 k \kappa\left(\phi \phi_{\omega}\right)^{1-p-q} \sqrt{r^{n}}\left(u_{1,0}^{n}\right)^{p-1}\left(u_{3,0}^{n}\right)^{q}+\mathcal{O}\left(k h^{2}\right),
$$

and from (76) and (81), taking into account that $-1<z_{j}<0$, one gets

$$
a_{1, j}^{n}>\frac{k}{h^{2}}\left(\frac{D_{1}}{4 r^{n}}-\frac{h}{2} \kappa\left(\phi \phi_{\omega}\right)^{1-p-q} \frac{\left(u_{1,0}^{n}\right)^{p-1}\left(u_{3,0}^{n}\right)^{q}}{\sqrt{r^{n}}}+\mathcal{O}\left(h^{3}\right)\right) .
$$

Note that the time horizon $T$ is chosen so that the carbonation front does not reach the sealed boundary, $L-\sqrt{r^{n}}>0, T=N k, 0 \leq n \leq N$. At any time $0 \leq t^{n} \leq T, 0 \leq n \leq N$, the carbonation front takes the value $\sqrt{r^{n}}$ between the initial and the final position. This value $\sqrt{r^{n}}$ does not reach the sealed boundary. Thus, there exists a positive number $\beta$ such that $\sqrt{r^{0}} / L<\beta<1$, verifying

$$
0<\sqrt{r^{0}}<\sqrt{r^{n}}<\sqrt{r^{N}} \leq \beta L<L
$$

Hence, using the induction hypothesis (106) for $i=1, i=3$ and $j=0$, and expression (109), coefficients $a_{1, j}^{n}$ in equation (108) are positive for $-M+1 \leq$ $j \leq-1$, with the condition on the spatial step size

$$
h_{1}<\frac{D_{1}}{2 \beta L \kappa\left(\phi \phi_{\omega}\right)^{1-p-q}\left(\tilde{G}_{1}\right)^{p-1}\left(\tilde{G}_{3}\right)^{q}} .
$$

For $i=2$, using (67) and (100), it follows that

$$
r^{n+1}-r^{n}=3 D_{2} \frac{k}{h}+\mathcal{O}\left(k h^{2}\right)
$$

and from (76) and (81), using that $-1<z_{j}<0$,

$$
a_{2, j}^{n}>\frac{k}{h^{2}}\left(\frac{D_{2}}{4 r^{n}}+\mathcal{O}\left(h^{3}\right)\right)
$$

and coefficients $a_{2, j}^{n}$ are positive for $-M+1 \leq j \leq-1$. 
And finally, for $i=5$, using (67) and (100), it follows that

$$
r^{n+1}-r^{n}=3 D_{5} \frac{k}{h}-2 k \kappa\left(\phi \phi_{\omega}\right)^{1-p-q} \sqrt{r^{n}} \frac{\left(u_{1,0}^{n}\right)^{p}\left(u_{3,0}^{n}\right)^{q}}{u_{5,0}^{n}}+\mathcal{O}\left(k h^{2}\right),
$$

and from (76) and (81), using that $-1<z_{j}<0$,

$$
a_{5, j}^{n}>\frac{k}{h^{2}}\left(\frac{D_{5}}{4 r^{n}}+\frac{h}{2} \kappa\left(\phi \phi_{\omega}\right)^{1-p-q} \frac{\left(u_{1,0}^{n}\right)^{p}\left(u_{3,0}^{n}\right)^{q}}{\sqrt{r^{n}} u_{5,0}^{n}}+\mathcal{O}\left(h^{3}\right)\right) .
$$

Hence, coefficients $a_{5, j}^{n}$ are positive for $-M+1 \leq j \leq-1$.

Now, regarding the concentrations of the chemical species in the uncarbonated zone, the positivity of the coefficients $a_{i, j}^{n}, i \in\{3,6\}$, in equations (76)(777) and (78) will be probed in analogous way using the value of the difference $r^{n+1}-r^{n}$ that appears in the transmission conditions (68).

For $i=3$, using (68) and (103), it follows that

$$
r^{n+1}-r^{n}=3 D_{3} \frac{k}{h} \frac{\sqrt{r^{n}}}{L-\sqrt{r^{n}}}+2 k \kappa\left(\phi \phi_{\omega}\right)^{1-p-q} \sqrt{r^{n}}\left(u_{1,0}^{n}\right)^{p}\left(u_{3,0}^{n}\right)^{q-1}+\mathcal{O}\left(k h^{2}\right),
$$

and from (777) and (81), using that $0<z_{j} \leq 1$,

$$
a_{3, j}^{n}>\frac{k}{h^{2}}\left(\frac{D_{3}}{4\left(L-\sqrt{r^{n}}\right)^{2}}-\frac{h}{2} \kappa\left(\phi \phi_{\omega}\right)^{1-p-q} \frac{\left(u_{1,0}^{n}\right)^{p}\left(u_{3,0}^{n}\right)^{q-1}}{\left(L-\sqrt{r^{n}}\right)}+\mathcal{O}\left(h^{3}\right)\right) .
$$

Note that the last expression (116), in an analogous way to the equation (108), presents a negative term, and positivity of (116), for $1 \leq j \leq M$, is guaranteed using the same arguments regarding the boundedness of $u_{1,0}^{n}$ and $u_{3,0}^{n}$, that was proved above for each time level $n$, with the following condition on the spatial step size

$$
h_{3}<\frac{D_{3}}{2\left(L-\sqrt{r^{0}}\right) \kappa\left(\phi \phi_{\omega}\right)^{1-p-q}\left(\tilde{G}_{1}\right)^{p}\left(\tilde{G}_{3}\right)^{q-1}} .
$$

Finally, for $i=6$, taking into account (68) and (103), it follows that

$$
r^{n+1}-r^{n}=3 D_{6} \frac{k}{h} \frac{\sqrt{r^{n}}}{L-\sqrt{r^{n}}}+\mathcal{O}\left(k h^{2}\right),
$$


and from (77) and (81), using that $0<z_{j} \leq 1$,

$$
a_{6, j}^{n}>\frac{k}{h^{2}}\left(\frac{D_{6}}{4\left(L-\sqrt{r^{n}}\right)^{2}}+\mathcal{O}\left(h^{3}\right)\right)
$$

and coefficients $a_{6, j}^{n}$ are positive for $1 \leq j \leq M$.

In conclusion, from (110) and (117), coefficients $a_{i, j}^{n}, 1 \leq i \leq 6, i \neq 4$, are positive under the condition on the spatial step size

$$
h<h_{0}=\min \left\{h_{1}, h_{3}\right\} .
$$

Since $0<r^{0}<r^{n}$, the nonnegativity of the coefficients $b_{i, j}^{n}, i \in\{1,2,5\}$, see (79), of the explicit scheme (76) is guaranteed, independently of the value of $n$, under the following respective conditions between the step sizes $h$ and $k$

$$
k_{1} \leq \frac{h^{2} r^{0}}{2 D_{1}+h^{2} r^{0} P_{1}}, \quad k_{2} \leq \frac{h^{2} r^{0}}{2 D_{2}+h^{2} r^{0} P_{2} Q_{2}}, \quad k_{5} \leq \frac{h^{2} r^{0}}{2 D_{5}}, \quad i \in\{1,2,5\} .
$$

The coefficients related to the uncarbonated zone $b_{i, j}^{n}, i \in\{3,6\}$, see (79), of the explicit scheme (77) are non negative, independently of the value of $n$, under the following conditions between $h$ and $k$

$$
k_{3} \leq \frac{h^{2} L^{2}(1-\beta)^{2}}{2 D_{3}+h^{2} L^{2}(1-\beta)^{2} S_{3, \mathrm{diss}}}, \quad k_{6} \leq \frac{h^{2} L^{2}(1-\beta)^{2}}{2 D_{6}}, \quad i \in\{3,6\} .
$$

Then, coefficients $b_{i, j}^{n}, 1 \leq i \leq 6, i \neq 4$, are positive under the condition

$$
k<k_{0}=\min \left\{k_{i}\right\}, \quad 1 \leq i \leq 6, \quad i \neq 4 .
$$

Consequently, from previous comments and induction argument, the numerical solution at time level $n+1$ is non negative, $u_{i, j}^{n+1} \geq 0, i \neq 4,-M \leq j \leq M$, 185 under conditions (120) and (123). Now, let us study the boundedness of the numerical solution.

Using (100) and (103), it holds

$$
\begin{aligned}
& u_{i, 0}^{n}<\frac{2}{3} u_{i,-1}^{n} \leq \tilde{G}_{i}, \quad i \in\{1,2,5\}, \\
& u_{i, 0}^{n}<\frac{2}{3} u_{i, 1}^{n} \leq \tilde{G}_{i}, \quad i \in\{3,6\} .
\end{aligned}
$$


Hence, from (76)-(77) and (78)-(80), together with assumptions (94) and (95), one gets

$$
\begin{aligned}
& u_{1, j}^{n+1} \leq\left(a_{1, j}^{n}+b_{1, j}^{n}+c_{1, j}^{n}\right) \tilde{G}_{1}+k P_{1} Q_{1} \tilde{G}_{2}=\left(1-k P_{1}\right) \tilde{G}_{1}+k P_{1} Q_{1} \tilde{G}_{2} \\
& \leq \tilde{G}_{1}, \quad-M+1 \leq j \leq-1, \\
& u_{2, j}^{n+1} \leq\left(a_{2, j}^{n}+b_{2, j}^{n}+c_{2, j}^{n}\right) \tilde{G}_{2}+k P_{2} \tilde{G}_{1}=\left(1-k P_{2} Q_{2}\right) \tilde{G}_{2}+k P_{2} \tilde{G}_{1} \\
& \leq \tilde{G}_{2}, \quad-M+1 \leq j \leq-1, \\
& \quad u_{3, j}^{n+1} \leq\left(a_{3, j}^{n}+b_{3, j}^{n}+c_{3, j}^{n}\right) \tilde{G}_{1}+k S_{3, \mathrm{diss}} u_{3, \mathrm{eq}}^{n} \\
& \quad=\left(1-k S_{3, \mathrm{diss}}\right) \tilde{G}_{3}+k S_{3, \mathrm{diss}} u_{3, \mathrm{eq}}^{n} \leq \tilde{G}_{3}, \quad 1 \leq j \leq M, \\
& u_{5, j}^{n+1} \leq\left(a_{5, j}^{n}+b_{5, j}^{n}+c_{5, j}^{n}\right) \tilde{G}_{5}=\tilde{G}_{5}, \quad-M+1 \leq j \leq-1, \\
& \quad u_{6, j}^{n+1} \leq\left(a_{6, j}^{n}+b_{6, j}^{n}+c_{6, j}^{n}\right) \tilde{G}_{6}=\tilde{G}_{6}, \quad 1 \leq j \leq M .
\end{aligned}
$$

From the induction proof for $u_{1,0}^{n}$ and $u_{3,0}^{n}$, and their bounds, we have

$$
0<u_{1,0}^{n}<\frac{2}{3} \tilde{G}_{1}, \quad 0<u_{3,0}^{n}<\frac{2}{3} \tilde{G}_{3} .
$$

From (82) and (130), taking into account the initial value $u_{4}^{0}=U_{40}$,

$$
u_{4}^{n}>u_{4}^{n-1} ; u_{4}^{n} \leq U_{40}+\kappa T\left(\phi \phi_{\omega}\right)^{1-p-q}\left(\frac{2}{3} \tilde{G}_{1}\right)^{p}\left(\frac{2}{3} \tilde{G}_{3}\right)^{q}, 1 \leq n \leq N .
$$

Using a mathematical induction argument and summarizing, under hypotheses (94)-(97), the following theorem shows that the numerical solution of problem (8)-(22), obtained from the scheme (76)-(77) and (82), preserves the qualitative properties satisfied by the theoretical solution obtained in Section 3 of [17]:

Theorem 1. Under hypotheses (94)-97), for small enough values of the step size $h$, verifying (110), (117) and (120), together with the step sizes conditions (121)-(123), the following conclusions hold true at the mesh points of the numerical domain: 
i) Approximate concentrations $u_{i, j}^{n}, i \in\{1,2,5\}$ of the scheme (76) in the carbonated zone are positive and uniformly bounded,

$$
0 \leq u_{i, j}^{n} \leq \tilde{G}_{i}, \quad-M \leq j \leq-1, \quad 0 \leq n \leq N
$$

ii) Approximate concentrations $u_{i, j}^{n}, i \in\{3,6\}$ of the scheme (77) in the uncarbonated region and uniformly bounded are positive,

$$
0 \leq u_{i, j}^{n} \leq \tilde{G}_{i}, \quad 1 \leq j \leq M, 0 \leq n \leq N
$$

iii) The solution $u_{4}^{n}$ of the scheme (82) for the calcium carbonate concentration is positive, increasing and bounded,

$$
u_{4}^{n} \leq U_{40}+\kappa T\left(\phi \phi_{\omega}\right)^{1-p-q}\left(\frac{2}{3} \tilde{G}_{1}\right)^{p}\left(\frac{2}{3} \tilde{G}_{3}\right)^{q}, 0 \leq n \leq N .
$$

iv) Approximate concentrations $u_{i, 0}^{n}, 1 \leq i \leq 6, i \neq 4$ at the carbonation front are positive and uniformly bounded for $0 \leq n \leq N$.

v) The carbonation front is positive and increasing, $0<r^{0}<r^{1}<\ldots<r^{N}$.

As a consequence of the boundedness of the mass concentrations, the stability of the numerical solution is also proved. For this purpose, let us denote the supremum norm of a vector $x=\left(x_{1}, x_{2}, \ldots, x_{n}\right)^{T}$ in $\mathbb{R}^{n}$ as $\|x\|_{\infty}=$ $\max \left(\left|x_{1}\right|,\left|x_{2}\right|, \ldots,\left|x_{n}\right|\right)$.

Amongst the many definitions of stability that exist in the literature, we will choose the following:

Definition 1. With previous notation, let us denote the vectors of concentrations $u_{i}^{n}=\left[u_{i,-M}^{n}, u_{i,-M+1}^{n}, \ldots, u_{i, 0}^{n}\right]^{T}, i \in\{1,2,5\}$, and $u_{i}^{n}=\left[u_{i, 0}^{n}, u_{i, 1}^{n}, \ldots, u_{i, M}^{n}\right]^{T}$, $i \in\{3,6\}$. We say that the numerical solution $\left\{u_{i, j}^{n}, 1 \leq i \leq 6, i \neq 4, u_{4}^{n}, 0 \leq\right.$ $n \leq N\}$ is $\|\cdot\|_{\infty}$-stable if there exist positive constants $C_{i}, 1 \leq i \leq 6$, independent of $n, k$ and $h$, such that

$$
\left\|u_{i}^{n}\right\|_{\infty} \leq C_{i}, \quad 1 \leq i \leq 6, \quad 0 \leq n \leq N .
$$


Thus, scheme (76)-(82) is $\|\cdot\|_{\infty}$-stable under assumptions (94)-(97), with the step sizes conditions (110), (117) and (120), together with (121)-(123), by taking

$$
C_{i}=\tilde{G}_{i}, \quad 1 \leq i \leq 6, \quad i \neq 4,
$$

and

$$
C_{4}=U_{40}+\kappa T\left(\phi \phi_{\omega}\right)^{1-p-q}\left(\frac{2}{3} \tilde{G}_{1}\right)^{p}\left(\frac{2}{3} \tilde{G}_{3}\right)^{q}
$$

Note that the results of Theorem 1 are conditioned to the step sizes restrictions stated there. The following example illustrates that these conditions can not be removed. Example 1. Consider the carbonation model (8)-(22) with parameters listed in the Table 1, see [16], with partial reaction orders $p=1.0$, $q=1.0$ and time horizon $T=1$ year, we obtain $h_{0}=0.0041$ and $k_{0}=0.0089$. ${ }_{210}$ Taking step sizes with values $h=0.05$ and $k=0.0106$, the positivity condition is broken. Figure 1 shows that positivity does not hold. Units in $\mathrm{x}$-axis are taken in $\mathrm{cm}$ and $\mathrm{y}$-axis in $\mathrm{g} \mathrm{cm}^{-3}$. 


\begin{tabular}{|c|c|}
\hline Magnitude & Value \\
\hline \multicolumn{2}{|c|}{ Initial concentrations $\left(\mathrm{g} \mathrm{cm}^{-3}\right)$} \\
\hline $\bar{U}_{10}(x), \quad 0<x<S_{0}$ & 0.0020 \\
\hline $\bar{U}_{20}(x), \quad 0<x<S_{0}$ & 0.0016 \\
\hline $\bar{U}_{30}(x), \quad S_{0}<x<L$ & 0.0120 \\
\hline $\bar{U}_{40}$ & 0.0000 \\
\hline $\bar{U}_{50}(x), \quad 0<x<S_{0}$ & 0.0050 \\
\hline $\bar{U}_{60}(x), \quad S_{0}<x<L$ & 0.0050 \\
\hline \multicolumn{2}{|c|}{ Exposed boundary concentrations $\left(\mathrm{g} \mathrm{cm}^{-3}\right)$} \\
\hline$\Lambda_{1}(t)$ & 0.0020 \\
\hline$\Lambda_{2}(t)$ & 0.0016 \\
\hline$\Lambda_{5}(t)$ & 0.0050 \\
\hline \multicolumn{2}{|c|}{ Equilibrium concentration of $\mathrm{Ca}(\mathrm{OH})_{2}\left(\mathrm{~g} \mathrm{~cm}^{-3}\right)$} \\
\hline$U_{3, \text { eq }}$ & 0.0050 \\
\hline \multicolumn{2}{|c|}{ Diffusion constants $\left(\mathrm{cm}^{2}\right.$ day $\left.^{-1}\right)$} \\
\hline$D_{1}$ & 0.62 \\
\hline$D_{2}$ & 3.50 \\
\hline$D_{3}$ & 0.86 \\
\hline$D_{5}$ & 1.00 \\
\hline$D_{6}$ & 1.00 \\
\hline \multicolumn{2}{|l|}{ Model parameters } \\
\hline$\alpha$ & 12500 \\
\hline$\kappa\left(\right.$ year $\left.^{-1}\right)$ & 750 \\
\hline$\phi$ & 0.10 \\
\hline$\phi_{\omega}$ & 0.50 \\
\hline$\phi_{a}$ & 0.50 \\
\hline$P_{1}=P_{2}\left(\right.$ day $\left.^{-1}\right)$ & 0.025 \\
\hline$Q_{1}=Q_{2}$ & 1.250 \\
\hline$S_{3, \text { diss }}\left(\right.$ day $\left.^{-1}\right)$ & 0.0075 \\
\hline \multicolumn{2}{|c|}{ Sample length and bounds of the carbonation depth } \\
\hline$L(\mathrm{~cm})$ & 12.00 \\
\hline$S_{0}(\mathrm{~cm})$ & 5.00 \\
\hline$\beta$ & 0.70 \\
\hline
\end{tabular}

Table 1: Data for numerical examples. 


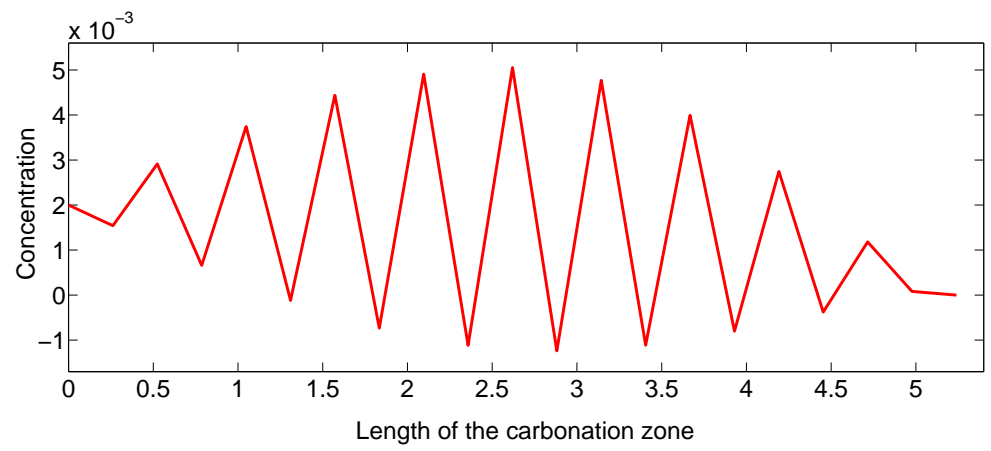

Figure 1: Numerical solution $\bar{U}_{1}(x, t)$ of Example 1 for $t=0.95$ years, when positivity condition is broken.

Next example illustrates the positivity and $\|\cdot\|_{\infty}$-stability of the solutions when conditions (110), (117) and (120), together with (121)-(123) are satisfied.

Example 2. With notation and model parameters listed in the Table 1, with $p=1.5, q=1.0$ and time horizon $T=10$ years, we get $h_{0}=0.0917$ and $k_{0}=0.0089$. Choosing step sizes $h=0.05$ and $k=0.005$, the positivity and stability of the solutions are guaranteed by Theorem 1, as Figure 2 shows. Units in $\mathrm{x}$-axis are taken in $\mathrm{cm}$ and $\mathrm{y}$-axis in $\mathrm{g} \mathrm{cm}^{-3}$. Furthermore, taking these step size values, the inequality (92) is satisfied, since $L=12 \mathrm{~cm}, \rho_{1}=-1.3120$ and the numerical carbonation front $\sqrt{r^{n}}$ measured in $\mathrm{cm}$ together with the nondimensional $\rho_{2, n}$ take the values shown in Figure 3. Thus, nonlinear equation (70) for $u_{3,0}^{n}$ is solvable and Algorithm 1 can be initiated. Units in x-axis are taken in years and y-axis in $\mathrm{cm}$. Table 2 shows CPU time taken in calculations for $h=0.05$ and several values of $k$, using MATLAB R2017b on processor Intel(R) Core(TM) i3-3110M CPU 2.40GHz. 

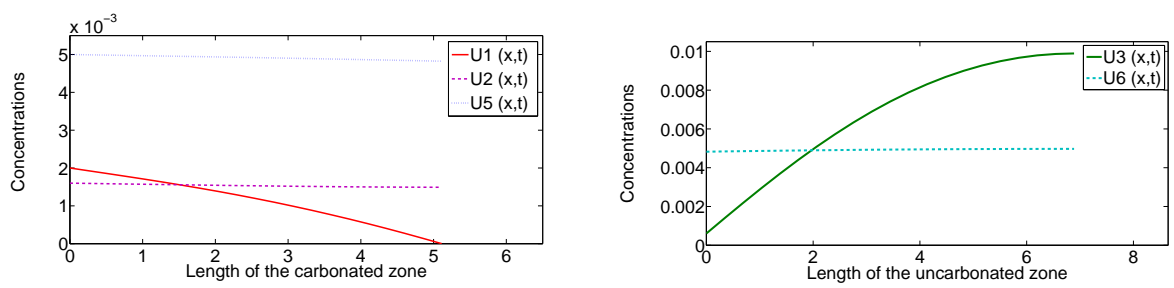

Figure 2: Numerical concentrations $\bar{U}_{i}(x, t), i \in\{1,2,5\}$, and $\bar{U}_{i}(x, t), i \in\{3,6\}$, in Example 2 for $t=9$ years, under stability conditions (110), (117) and (120), (121)-(123) .
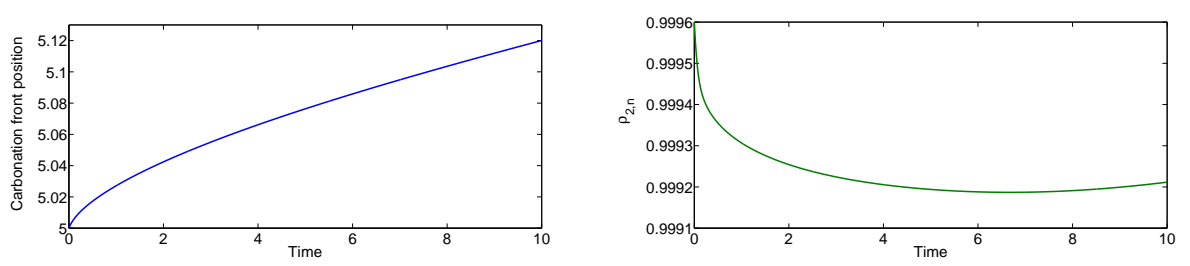

Figure 3: Position of the numerical carbonation front $\sqrt{r^{n}}$, and values of $\rho_{2, n}$, in Example 2, as a function of time.

\begin{tabular}{lcccc}
\hline \hline$k$ & 0.008 & 0.006 & 0.004 & 0.002 \\
\hline$\sqrt{r^{N}}(\mathrm{~cm})$ & 5.1200 & 5.1200 & 5.1201 & 5.1201 \\
\hline CPU time $(\mathrm{s})$ & 1.081 & 1.676 & 2.683 & 6.899 \\
\hline \hline
\end{tabular}

Table 2: Dependence of process time on the temporal step size.

\section{Monotonicity of the numerical solution}

In this section, we present monotone properties of the numerical solution of the scheme (76)-(77), according to the following definition, see [7]:

Definition 2. Let $F\left(w_{j}^{n}\right)=0$ be a numerical scheme, where index $n$ refers to the time and $j$ to the space, $j \in \mathcal{J}, n \in \mathcal{N}$. We say that the numerical scheme $F\left(w_{j}^{n}\right)=0$ is spatial monotone time preserving if, assuming that $w^{n}$ is a spatial monotone sequence at time level $n$, then so is $w^{n+1}$ for all time index $n$. 
We will state that the scheme (76)-(77) preserves the monotone decreas235 ing spatial behaviour of the numerical concentrations $u_{i}^{n}, i \in\{1,2,5\}$, in the carbonated zone, while the concentrations $u_{i}^{n}, i \in\{3,6\}$, in the uncarbonated zone are monotone increasing in space, for all time level $n$. We will prove this property using the induction principle on the temporal index $n$.

Firstly, we state that, under the positivity constraints (110), (117) and (120), together with (121)-(123), and assuming

$$
u_{i, j+1}^{n} \leq u_{i, j}^{n}, \quad-M+1 \leq j \leq-2, \quad i \in\{1,2,5\},
$$

then, the numerical solution at time level $n+1$ satisfies

$$
u_{i, j+1}^{n+1} \leq u_{i, j}^{n+1}, \quad-M+1 \leq j \leq-2, \quad i \in\{1,2,5\} .
$$

Let us start by considering $i=1$. From (76) and (138) one gets

$$
\begin{aligned}
& u_{1, j}^{n+1} \geq a_{1, j}^{n} u_{1, j}^{n}+b_{1, j}^{n} u_{1, j}^{n}+c_{1, j}^{n} u_{1, j+1}^{n}+k P_{1} Q_{1} u_{2, j}^{n} \\
& =\left(1-\frac{D_{1} k}{h^{2} r^{n}}-\left(\frac{1+z_{j}}{4 h}\right) \Delta_{1}^{n}-k P_{1}\right) u_{1, j}^{n}+\left(\frac{D_{1} k}{h^{2} r^{n}}+\left(\frac{1+z_{j}}{4 h}\right) \Delta_{1}^{n}\right) u_{1, j+1}^{n} \\
& +k P_{1} Q_{1} u_{2, j}^{n}, \quad-M+1 \leq j \leq-2
\end{aligned}
$$

and

$$
\begin{aligned}
& u_{1, j+1}^{n+1} \leq a_{1, j+1}^{n} u_{1, j}^{n}+b_{1, j+1}^{n} u_{1, j+1}^{n}+c_{1, j+1}^{n} u_{1, j+1}^{n}+k P_{1} Q_{1} u_{2, j+1}^{n} \\
& =\left(\frac{D_{1} k}{h^{2} r^{n}}-\left(\frac{1+z_{j+1}}{4 h}\right) \Delta_{1}^{n}\right) u_{1, j}^{n}+\left(1-\frac{D_{1} k}{h^{2} r^{n}}+\left(\frac{1+z_{j+1}}{4 h}\right) \Delta_{1}^{n}-k P_{1}\right) u_{1, j+1}^{n} \\
& +k P_{1} Q_{1} u_{2, j+1}^{n}, \quad-M+1 \leq j \leq-2
\end{aligned}
$$

Using the positivity of coefficient $b_{1, j}^{n}$ and $\Delta_{1}^{n}$ shown in Theorem 1 from (79) for $i=1$, (138) and (140)-(141), it follows that

$$
\begin{aligned}
& u_{1, j+1}^{n+1}-u_{1, j}^{n+1} \leq\left(b_{1, j}^{n}+\frac{1}{4} \Delta_{1}^{n}\right)\left(u_{1, j+1}^{n}-u_{1, j}^{n}\right) \\
& +k P_{1} Q_{1}\left(u_{2, j+1}^{n}-u_{2, j}^{n}\right) \leq 0,-M+1 \leq j \leq-2,
\end{aligned}
$$


and then

$$
u_{1, j+1}^{n+1} \leq u_{1, j}^{n+1} .
$$

For the sake of brevity and because of the analogy of the cases $i=2$ and $240 \quad i=5$, we omit the proofs of the decreasing monotone behaviour of $w_{2}^{n}$. and $w_{5, \cdot}^{n}$.

Regarding the concentrations in the uncarbonated zone $u_{i, j}^{n}, i \in\{3,6\}$, we will prove that spatial increasing monotonicity requires some additional restrictions on the step sizes discretizations. Thus, under these additional restrictions, and the positivity conditions (110), (117) and (120), together with (121)-(123), if we assume

$$
u_{i, j+1}^{n} \geq u_{i, j}^{n}, \quad 1 \leq j \leq M-1, \quad i \in\{3,6\},
$$

then, the numerical solution at time level $n+1$ satisfies

$$
u_{i, j+1}^{n+1} \geq u_{i, j}^{n+1}, \quad 1 \leq j \leq M-1, \quad i \in\{3,6\} .
$$

Let us start by considering the case $i=3$. From (77) and (144), one gets

$$
\begin{aligned}
& u_{3, j}^{n+1} \leq a_{3, j}^{n} u_{3, j}^{n}+b_{3, j}^{n} u_{3, j}^{n}+c_{3, j}^{n} u_{3, j+1}^{n}+k S_{3, \mathrm{diss}} u_{3, \mathrm{eq}}^{n} \\
& =\left(1-\frac{D_{3} k}{h^{2} \Delta_{3}^{n}}+\left(\frac{z_{j}-1}{4 h}\right) r^{n} \frac{\Delta_{1}^{n} \Delta_{2}^{n}}{\Delta_{3}^{n}}-k S_{3, \mathrm{diss}}\right) u_{3, j}^{n} \\
& +\left(\frac{D_{3} k}{h^{2} \Delta_{3}^{n}}+\left(\frac{1-z_{j}}{4 h}\right) r^{n} \frac{\Delta_{1}^{n} \Delta_{2}^{n}}{\Delta_{3}^{n}}\right) u_{3, j+1}^{n}+k S_{3, \mathrm{diss}} u_{3, \mathrm{eq}}^{n}, \quad 1 \leq j \leq M-1,
\end{aligned}
$$

and

$$
\begin{aligned}
& u_{3, j+1}^{n+1} \geq a_{3, j+1}^{n} u_{3, j}^{n}+b_{3, j+1}^{n} u_{3, j+1}^{n}+c_{3, j+1}^{n} u_{3, j+1}^{n}+k S_{3, \mathrm{diss}} u_{3, \mathrm{eq}}^{n} \\
& =\left(\frac{D_{3} k}{h^{2} \Delta_{3}^{n}}+\left(\frac{z_{j+1}-1}{4 h}\right) r^{n} \frac{\Delta_{1}^{n} \Delta_{2}^{n}}{\Delta_{3}^{n}}\right) u_{3, j}^{n} \\
& +\left(1-\frac{D_{3} k}{h^{2} \Delta_{3}^{n}}+\left(\frac{1-z_{j+1}}{4 h}\right) r^{n} \frac{\Delta_{1}^{n} \Delta_{2}^{n}}{\Delta_{3}^{n}}-k S_{3, \mathrm{diss}}\right) u_{3, j+1}^{n}+k S_{3, \mathrm{diss}} u_{3, \mathrm{eq}}^{n}, \quad 1 \leq j \leq M-1 .
\end{aligned}
$$


Using the positivity of $b_{3, j}^{n}$ and $\Delta_{1}^{n}$ shown in Theorem 1 from (79) for $i=3$, (138) and (140)-(141), it follows that

$$
u_{3, j+1}^{n+1}-u_{3, j}^{n+1} \geq\left(b_{3, j}^{n}-\frac{r^{n}}{4} \frac{\Delta_{1}^{n} \Delta_{2}^{n}}{\Delta_{3}^{n}}\right)\left(u_{3, j+1}^{n}-u_{3, j}^{n}\right) \geq 0, \quad 1 \leq j \leq M-1 .
$$

Note that the left hand side of expression (148) is positive if the bracket coefficient is also positive. From (72), (81) and (109), one gets

$$
\frac{r^{n}}{4} \frac{\Delta_{1}^{n} \Delta_{2}^{n}}{\Delta_{3}^{n}}=\frac{r^{n+1}-r^{n}}{4 \sqrt{r^{n}}\left(L-\sqrt{r^{n}}\right)} \leq \frac{k \alpha \kappa\left(\phi \phi_{\omega}\right)^{1-p-q} \tilde{G}_{1}^{p} \tilde{G}_{3}^{q-1}}{2 L(1-\beta)} .
$$

Thus, from (79) for $i=3$, (148) and (149), the difference $u_{3, j+1}^{n+1}-u_{3, j}^{n+1}$ is non negative under the following requirement linking spatial and temporal step sizes:

$$
k \leq k_{3}^{*}=\frac{2 h^{2} L^{2}(1-\beta)^{2}}{4 D_{3}+2 h^{2} L^{2}(1-\beta)^{2} S_{3, \mathrm{diss}}+h^{2} L(1-\beta) \alpha \kappa\left(\phi \phi_{\omega}\right)^{1-p-q} \tilde{G}_{1}^{p} \tilde{G}_{3}^{q-1}} .
$$

Finally, for the case $i=6$, using similar arguments, it is easy to show that $u_{6,}^{n}$. presents a spatial increasing monotone behaviour under the additional condition on the relation between spatial and temporal step sizes:

$$
k \leq k_{6}^{*}=\frac{2 h^{2} L^{2}(1-\beta)^{2}}{4 D_{6}+h^{2} L(1-\beta) \alpha \kappa\left(\phi \phi_{\omega}\right)^{1-p-q} \tilde{G}_{1}^{p} \tilde{G}_{3}^{q-1}} .
$$

Summarizing, the following result can be established:

Theorem 2. Under hypotheses 947- 977), assuming the positivity conditions (120) and (123), and the temporal step size conditions (150) and (151), the 245 numerical scheme (76)-(82) is spatial monotone preserving in the sense of Definition 圆.

Consequently, starting with $u_{i, \cdot}^{0}$ spatial monotone decreasing sequences, $i \in$ $\{1,2,5\}$, the numerical solution remains monotone decreasing for all $1 \leq n \leq$

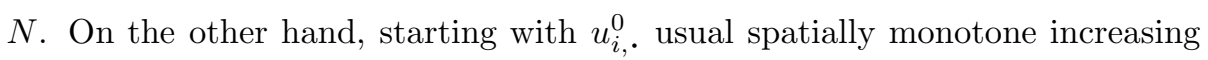


sequences, $i \in\{3,6\}$, the numerical solution remains monotone increasing for all $n$. Note that positive conditions are not satisfied in Example 1 and then monotonicity is not guaranteed as it can be seen in Figure 1. Next Example 3 illustrates the monotone behaviour under constraints of Theorem 2 .

Example 3. With data of Table 1, taking reaction orders $p=1.5, q=1$ 255 and time horizon $T=10$ years, with previous notation, we obtain $h_{0}=0.0917$, $k_{0}=0.0089, k_{3}^{*}=0.0059$ and $k_{6}^{*}=0.0056$. Choosing step sizes $h=0.05$ and $k=0.005$ satisfying the monotonicity requirements of Theorem [2, Figures 3 and 4 show the monotone behaviour of the numerical solutions of $\bar{U}_{1}(x, t)$ and $\bar{U}_{3}(x, t)$ for several equidistant fixed values of time.

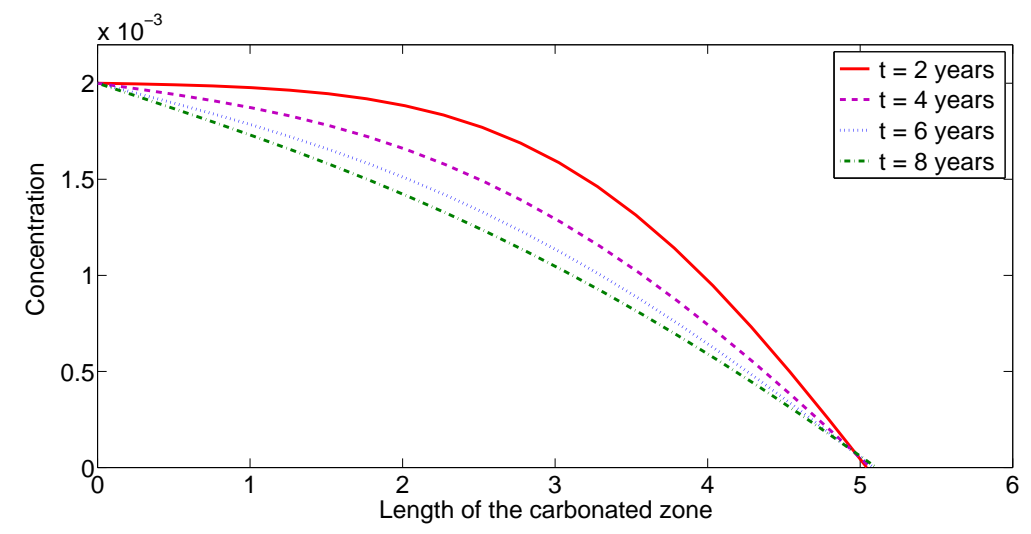

Figure 4: Numerical solution $\bar{U}_{1}(x, t)$ of Example 3, for several equidistant times.

\section{Conclusions}

From the applications point of view, a theoretical model needs to be checked numerically. In this paper, we construct reliable numerical solutions of the concrete carbonation model proposed in [16] and [17]. In fact, we show that the proposed numerical solutions are positive and preserve qualitative properties of the theoretical solution such as concentrations boundedness. One the advantages of our approach is that concentrations monotonicity properties suggested 


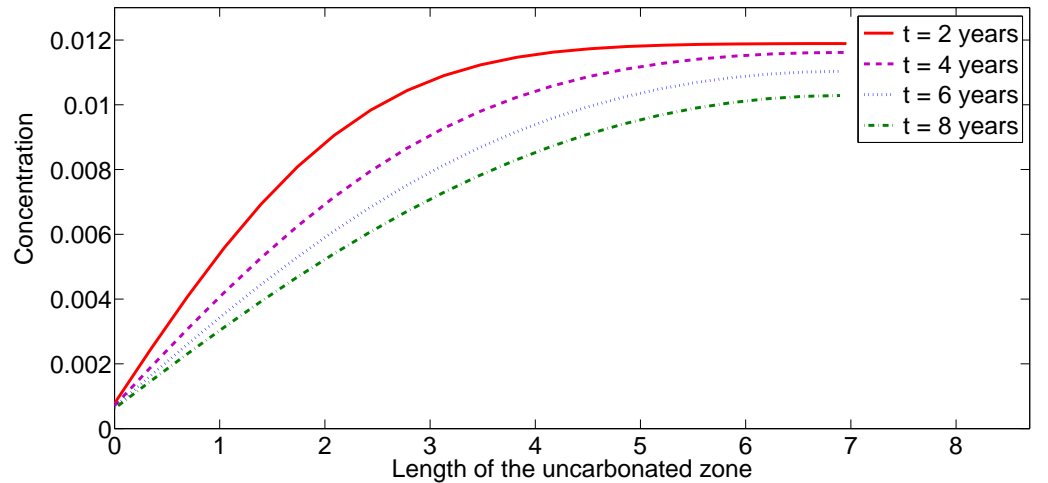

Figure 5: Numerical solution $\bar{U}_{3}(x, t)$ of Example 3, for several equidistant times.

in the experiments, although not proved theoretically in [17], are confirmed throughout the behaviour of the numerical solution and illustrated with numerical examples. The numerical analysis includes sufficient conditions on the step sizes discretization, explicitly given in terms of the data in order to satisfy the above properties.

\section{Acknowledgements}

This work has been partially supported by the Ministerio de Economía y Competitividad Spanish grant MTM 2017-89664-P .

\section{References}

[1] E. Bastidas-Arteaga, F. Schoefs, M.G. Stewart, X. Wang, "Influence of global warming on durability of corroding RC structures: A probabilistic approach", Engineering Structures, 51, 259-266, 2013.

[2] J. Bear, Dynamics of Fluids in Porous Media, Dover Publications Inc., New York, 1972.

[3] D. Bunte, Zum Karbonatisierungsbedingten Verlust der Dauerhaftigkeit von 
Auenbauteilen aus Stahlbeton, PhD Thesis, Technischen Universität Braunschweig, 1994.

[4] J. Crank, Free and Moving Boundary Problems, Oxford Science Publications, 1984.

[5] P. Danckwerts, Gas-liquid Reactions, McGraw-Hill Book Company, New York, 1970.

[6] T. Fatima, A. Muntean, "Sulfate attack in sewer pipes: Derivation of a concrete corrosion model via two-scale convergence", Nonlinear Analysis: Real World Applications, vol. 15, no. 1, pp. 326-344 (2014).

[7] S. K. Godunov, "A difference scheme for numerical solution of discontinuous solution of hydrodynamic equations", Math. Sbornik, 47, 271-306, 1959.

[8] IPCC. Climate Change 200\%: The Physical Science Basis. Contribution of Working Group I to the Fourth Assessment Report of the Intergovernmental Panel on Climate Change, Cambridge University Press, New York, 2007.

[9] T. Ishida, K. Maekawa, M. Soltani. "Theoretically identified strong coupling of carbonation rate and thermodynamic moisture states in micropores of concrete", Journal of Advanced Concrete Technology, 2 (2004), no. $2,213-222$.

[10] Y. Kishitani, S. Hokoi, K. Harada, S. Takada, "Prediction model for carbonation of concrete structures considering heat and moisture transfer", $J$. Structural and Construction Engineering Trans. AIJ, 595, 17-23 (2005).

[11] K. Kumazaki, "A mathematical model of carbon dioxide transport in concrete carbonation process", Discrete and Continuous Dynamical Systems Series $S 7$ (1), 113-125 (2014).

[12] H. G. Landau, "Heat condution in a melting solid", Quarterly of Applied Mathematics, vol. 8, pp. 81-95, 1950. 
[13] J. D. Logan, Transport Modeling in Hydrogeochemical Systems, volume 17 of Interdisciplinary Applied Mathematics, Springer Verlag, New York, 2001.

310

[14] M. Mainguy, O. Coussy, "Propagation Fronts during Calcium Leaching and Chloride Penetration", Journal of Engineering Mechanics, ASCE, 126 (3), 250-257 (2000).

[15] A. Muntean, A Moving-boundary Problem: Modeling, Analysis and Simulation of Concrete Carbonation, Cuvillier Verlag, Göttingen, 2006. PhD

[16] A. Muntean, M. Böhm, J. Kropp, "Moving carbonation fronts in concrete: A moving-sharp-interface approach", Chemical Engineering Science, 66 (2011), no. 3, 538-547.

[17] A. Muntean, M. Böhm, "A moving-boundary problem for concrete carbona-

[19] M.A. Piqueras, R. Company, L. Jódar, "A front-fixing numerical method for a free boundary nonlinear diffusion logistic population model", Journal of Computational and Applied Mathematics, 309 (2017), 473-481.

[20] A.A. Ramezanianpour, S.A. Ghahari, M. Esmaeili, "Effect of combined carbonation and chloride ion ingress by an accelerated test method on microscopic and mechanical properties of concrete", Construction and Building Materials, 58 (2014), 138-146.

[21] M. Tadi, "A four-step fixed-grid method for 1D Stefan problems", ASME J. Heat Transfer , 132(2010), 114502-114505. 
[22] H.F.W. Taylor, Cement Chemistry, Thomas Telford Publishing, London, 335 1997.

[23] K. Tuutti, Corrosion of steel in concrete, Swedish Cement and Concrete Research Institute (CBI), Stockholm, 1982.

[24] T. Uomoto, Y. Takada, "Factors affecting concrete carbonation rate", Durability of Building Materials Components, 6, 1133-1141 (1993).

340 [25] I.S. Yoon, "Deterioration of concrete due to combined reaction of carbonation and chloride penetration: Experimental study", Key Engineering Materials, vol. 348-349 (2007) pp. 729-732. 